\title{
Super RSK correspondence with symmetry
}

\author{
Robert Muth \\ Department of Mathematics \\ Washington \& Jefferson College \\ Washington, PA 15301, U.S.A. \\ rmuth@washjeff.edu
}

Submitted: Sep 5, 2018; Accepted: May 5, 2019; Published: May 17, 2019

(c) The author. Released under the CC BY-ND license (International 4.0).

\begin{abstract}
Super RSK correspondence is a bijective correspondence between superbiwords and pairs of semistandard supertableaux. Such a bijection was given by Bonetti, Senato and Venezia, via an insertion algorithm closely related to Schensted insertion. Notably, the symmetry property satisfied by the classical RSK bijection holds only in special cases under this bijection. We present a new super RSK bijection, based on the mixed insertion process defined by Haiman, where the symmetry property holds in complete generality.
\end{abstract}

Mathematics Subject Classifications: 05A19, 16T30

\section{Introduction}

The work of Robinson [R] in 1938, and Schensted [S] in 1961, describes a bijection between permutations and pairs of same-shape standard tableaux, now known as the RobinsonSchensted (RS) correspondence. A key ingredient in the bijection is an algorithm called Schensted insertion. In 1970, Knuth showed that Schensted insertion could be adapted to a more general setting to achieve a bijection between two-line arrays of letters called 'biwords' (which are in natural bijection with matrices of non-negative integers) and pairs of same-shape semistandard tableaux $[\mathrm{K}]$. This bijection is known as the RobinsonSchensted-Knuth (RSK) correspondence.

A celebrated feature of the RSK correspondence is a certain symmetry property; namely, exchanging the rows of a biword (or, transposing the matrix from the matrix perspective) translates via the RSK correspondence to exchanging the positions of the associated pair of semistandard tableaux. This property, proven for the RS correspondence by Viennot [V] in 1977 and extended to the full RSK correspondence by Fulton $[\mathrm{F}]$ in 1997, is far from obvious from the workings of the RSK algorithm itself. 
The RSK correspondence has applications in a variety of settings; of particular relevance for this paper is its application in representation theory and invariant theory, where it describes a bijection between various important bases for associative algebras and Lie algebras. We consider here the generalization of RSK correspondence to combinatorial objects associated with the representation theory and invariant theory of superalgebras. These 'super' combinatorial objects are restricted superbiwords and semistandard supertableaux. In contrast with the classical situation, letters in restricted superbiwords can have even or odd parity, with repetition of mixed-parity biletters disallowed. Semistandard supertableaux are nondecreasing tableaux in which letters of even parity strictly increase down columns, and letters of odd parity strictly increase along rows. See for example [CPT, DR, GRS, LNS, MZ] for a few instances of these combinatorial objects arising in the study of bases of superalgebras and their representations. In particular, 'super'-RSK correspondence plays a key role in the theory of generalized Schur algebras associated to quasihereditary superalgebras, as it provides a bijection between natural bases and heredity bases for these algebras, see [KM].

In this paper we prove that an adaptation of the mixed insertion algorithm defined by Haiman $[\mathrm{H}]$ can be used to define a super-RSK correspondence between restricted superbiwords and same-shape pairs of semistandard supertableaux. This correspondence fully generalizes the classical RSK correspondence, in the sense that classical RSK can be viewed as a specialization of super-RSK to the case of even-parity superbiwords, and the classical symmetry property described above holds for super-RSK in full generality.

Numerous variants of super-RSK correspondence exist in the literature. Most notably, Bonetti, Senato, and Venezia [BSV] presented a different correspondence between the same sets of combinatorial objects considered in this paper. At the heart of their algorithm are dual insertion processes which are very much like the classical Schensted insertion process, in that insertion progresses linearly from one row to the next (or one column to the next), and the number of 'bumps' in a given insertion is bounded by the number of rows (or the number of columns) in the Young diagram. By contrast, the Haiman insertion process utilized in this paper progresses in a less direct fashion, where the number of 'bumps' in an insertion is bounded only by the number of nodes in the Young diagram. A more crucial difference between the two algorithms is the fact that the super-RSK of [BSV] does not have the symmetry property in general (see Example 31). La Scala, Nardozza and Senato describe [LNS, Proposition 4.7] a subset of superbiwords where symmetry is known to hold for the [BSV] correspondence, but a complete description of such biwords is still an open problem.

Another variant of super-RSK correspondence appears in work by Shimozono and White [SW]. Their algorithm is based around the same Haiman insertion algorithm as used here, but adapted to work with a different class of combinatorial objects: unrestricted superbiwords (repetitions of mixed biletters allowed), and supertableaux which are row-weak and column-strict with respect to both parities. They demonstrate a bijection between these objects, and prove that their correspondence generalizes the classical symmetry property as well. While the [SW] algorithm generally yields different supertableaux from ours (see again Example 31), we note that they agree, crucially, in the special case 
of 'standard' superbiwords - those with no repeated letters. This is a key ingredient in the proof of the symmetry of our super-RSK correspondence.

Now for a description of the structure of this paper. In $\S 2$, we set up basic notation and definitions of the relevant combinatorial objects. In $\S 3$, we describe the $\varepsilon$-insertion process which drives the super-RSK algorithm, and prove some useful lemmas about the process. In $\S 4$, we prove some bounds on the distribution of bumped nodes during the insertion process, which are necessary for the results in the subsequent section, and perhaps of independent interest in the study of tableau growth. In $\S 5$, we define the super-RSK map 'sRSK' and prove the first main theorem of the paper, which appears as Theorem 21 in the text:

Theorem 1 (Super-RSK correspondence). The map sRSK defines a bijection between restricted superbiwords and same-shape pairs of semistandard supertableaux.

In $\S 6$, we prove some lemmas related to standardizing superbiwords, and prove the other main theorem of the paper, which appears as Corollary 30 in the text:

Theorem 2 (Super-RSK symmetry). Under super-RSK correspondence, exchanging rows in the superbiword $\boldsymbol{w}$ is equivalent to exchanging the positions of the pair of supertableaux $\operatorname{sRSK}(\boldsymbol{w})$.

\section{Preliminaries}

Since all combinatorial objects considered in this paper are $\mathbb{Z}_{2}$-colored, we will henceforth suppress the prefix 'super' from most of our terminology.

\subsection{Alphabets}

An alphabet $\mathscr{X}$ is a set equipped with a parity function $\mathscr{X} \rightarrow \mathbb{Z}_{2}, x \mapsto \bar{x}$, and a total order $<\mathscr{X}$. Elements of alphabets are called letters. We call $x \in \mathscr{X}$ even if $\bar{x}=\overline{0}$ and odd if $\bar{x}=\overline{1}$. Let $\prec \mathscr{X}$ be the total order on $\mathscr{X}$ defined by

$$
a \prec \mathscr{X} b \Longleftrightarrow\left\{\begin{array}{l}
\bar{a}=\overline{1}, \bar{b}=\overline{0}, \text { or } \\
\bar{a}=\bar{b}=\overline{0} \text { and } a<\mathscr{X} b, \text { or } \\
\bar{a}=\bar{b}=\overline{1} \text { and } a>_{\mathscr{X}} b .
\end{array}\right.
$$

Note then that

$$
a<\mathscr{X} b \Longrightarrow \begin{cases}a \prec \mathscr{X} b & \text { if } \bar{b}=\overline{0} \\ a \succ_{\mathscr{X}} b & \text { if } \bar{b}=\overline{1} .\end{cases}
$$

The dual alphabet $\mathscr{X}^{*}$ of an alphabet $\mathscr{X}$ has underlying set $\left\{x^{*} \mid x \in \mathscr{X}\right\}$, parity function defined by $\overline{x^{*}}=\bar{x}+\overline{1}$, and total order $<\mathscr{X}^{*}$ defined so that

$$
x^{*}<\mathscr{X}^{*} y^{*} \Longleftrightarrow x<x_{\mathscr{X}} y \text {. }
$$

THE ELECTRONiC JOURNAL Of COMBINATORICS 26(2) (2019), \#P2.27 
It follows that $a^{*} \prec \mathscr{X}^{*} b^{*}$ if and only if $a \succ \mathscr{X} b$.

The standardizing alphabet $\mathscr{X} \bullet$ of an alphabet $\mathscr{X}$ has underlying set $\left\{x^{(i)} \mid x \in \mathscr{X}, i \in\right.$ $\left.\mathbb{Z}_{>0}\right\}$, parity function defined by $\overline{x^{(i)}}=\bar{x}$, and total order $<_{\mathscr{X}} \bullet$ defined so that

$$
a^{(i)}<\mathscr{X} \bullet b^{(j)} \Longleftrightarrow\left\{\begin{array}{l}
a<\mathscr{X} b, \text { or } \\
a=b \text { and } i<j .
\end{array}\right.
$$

Define the 'forget superscripts' function $\hat{\bullet}: \mathscr{X}^{\bullet} \rightarrow \mathscr{X}$ by $x^{(i)} \mapsto x$.

Going forward, we will suppress the subscripts and write $<$ or $\prec$ when the underlying alphabet is clear from context.

\subsection{Tableaux}

We set $\mathbf{N}:=\mathbb{Z}_{>0} \times \mathbb{Z}_{>0}$ and refer to the elements of $\mathrm{N}$ as nodes. Define a partial order $\leqslant$ on $\mathbf{N}$ as follows: $(r, s) \leqslant\left(r^{\prime}, s^{\prime}\right)$ if and only if $r \leqslant r^{\prime}$ and $s \leqslant s^{\prime}$. For $u=(r, s) \in \mathbf{N}$ we will write $u^{\prime}:=(s, r) \in \mathbf{N}$.

For $\varepsilon \in \mathbb{Z}_{2}$ and $u=(r, s) \in \mathbf{N}$, define

$$
u_{\varepsilon}= \begin{cases}r & \varepsilon=\overline{0} \\ s & \varepsilon=\overline{1}\end{cases}
$$

and for $i \in \mathbb{Z}_{>0}$ define

$$
\mathrm{N}(\varepsilon, i)=\left\{u \in \mathrm{N} \mid u_{\varepsilon}=i\right\}
$$

I.e., $\mathrm{N}(\varepsilon, i)$ is the $i$ th row of nodes if $\varepsilon=\overline{0}$, and the $i$ th column of nodes if $\varepsilon=\overline{1}$.

We write $u \uparrow v$ if $u_{\overline{0}} \geqslant v_{\overline{0}}$ and $u_{\overline{1}}=v_{\overline{1}}$, and $u \Uparrow v$ if the inequality is strict. We write $u \nearrow v$ if $u_{\overline{0}} \geqslant v_{\overline{0}}$ and $u_{\overline{1}} \leqslant v_{\overline{1}}$, and $u \nearrow v$ if both inequalities are strict. We similarly define the symbols $\rightarrow, \Rightarrow, \searrow, \Downarrow, \downarrow, \Downarrow$.

For $n \in \mathbb{Z}_{\geqslant 0}$, we say $\lambda=\left(\lambda_{1}, \ldots, \lambda_{n}\right) \in \mathbb{Z}_{\geqslant 0}^{n}$ is a partition of $n$, writing $\lambda \vdash n$, if $\lambda_{1} \geqslant \cdots \geqslant \lambda_{n}$ and $\sum \lambda_{i}=n$. Let $\Lambda_{+}(n)$ be the set of all partitions of $n$. The Young diagram of $\lambda$ is

$$
[\lambda]=\left\{(r, s) \in \mathbf{N} \mid s \leqslant \lambda_{r}\right\}
$$

We say a node $u$ of $[\lambda]$ is removable if $[\lambda] \backslash\{u\}=[\mu]$ for some partition $\mu$. We say a node $u \notin[\lambda]$ is addable if $[\lambda] \cup\{u\}=[\mu]$ for some partition $\mu$. For a partition $\lambda$, the conjugate partition $\lambda^{\prime}$ is defined such that $u \in\left[\lambda^{\prime}\right]$ if and only if $u^{\prime} \in[\lambda]$.

An $(\mathscr{X}, \lambda)$-tableau is a function $\mathrm{T}:[\lambda] \rightarrow \mathscr{X}$. If $\mathrm{T}$ is an $(\mathscr{X}, \lambda)$-tableau, we write $\operatorname{sh}(\mathrm{T})=\lambda$. The content $\operatorname{con}(\mathrm{T})$ of an $(\mathscr{X}, \lambda)$-tableau $\mathrm{T}$ is the multiset $\{\mathrm{T}(u) \mid u \in[\lambda]\}$.

An $(\mathscr{X}, \lambda)$-tableau is semistandard if: 
(i) it is non-decreasing: $\mathrm{T}(u) \leqslant \mathscr{X} \mathrm{T}(v)$ whenever $u \leqslant v$.

(ii) it is row-strict with respect to odd letters: if $\mathrm{T}(u)=\mathrm{T}(v)$ for $u, v$ in the same row, then $\overline{\mathrm{T}(u)}=\overline{0}$.

(iii) it is column-strict with respect to even letters: if $\mathrm{T}(u)=\mathrm{T}(v)$ for $u, v$ in the same column, then $\overline{\mathrm{T}(u)}=\overline{1}$.

A standard tableau is a semistandard tableau such that $\mathrm{T}(u) \neq \mathrm{T}(v)$ for every $u \neq v \in$ $[\lambda]$. For an $(\mathscr{X}, \lambda)$-tableau $\mathrm{T}$, define the dual $\left(\mathscr{X}^{*}, \lambda\right)$-tableau $\mathrm{T}^{*}$ by $\mathrm{T}^{*}(u):=\mathrm{T}(u)^{*}$, and define the conjugate $\left(\mathscr{X}, \lambda^{\prime}\right)$-tableau $\mathrm{T}^{\prime}$ by $\mathrm{T}^{\prime}(u):=\mathrm{T}\left(u^{\prime}\right)$. We write $\mathrm{T}^{\prime *}:=\left(\mathrm{T}^{\prime}\right)^{*}=\left(\mathrm{T}^{*}\right)^{\prime}$ for the dual conjugate $\left(\mathscr{X}^{*}, \lambda^{\prime}\right)$-tableau. The following lemmas are obvious.

Lemma 1. The following are equivalent:

(i) $\mathrm{T}$ is a standard $(\mathscr{X}, \lambda)$-tableau.

(ii) $\mathrm{T}^{\prime}$ is a standard $\left(\mathscr{X}, \lambda^{\prime}\right)$-tableau.

(iii) $\mathrm{T}^{*}$ is a standard $\left(\mathscr{X}^{*}, \lambda\right)$-tableau.

Lemma 2. An $(\mathscr{X}, \lambda)$-tableau $\mathrm{T}$ is semistandard if and only if the dual conjugate $\mathrm{T}^{\prime *}$ is a semistandard $\left(\mathscr{X}^{*}, \lambda^{\prime}\right)$-tableau.

\subsection{Standardizing tableaux}

Recalling the standardizing alphabet $\mathscr{X}^{\bullet}$ from $\S 2.1$, for any $(\mathscr{X} \bullet, \lambda)$-tableau $\mathrm{T}$, define $\hat{\bullet}(\mathrm{T}):=\hat{\bullet} \circ \mathrm{T}$. I.e., $\hat{\bullet}(\mathrm{T})$ is the tableau $\mathrm{T}$ with superscripts deleted. We say a standard $(\mathscr{X} \bullet, \lambda)$-tableau $\mathrm{U}$ is $\bullet$-standard provided

(i) $\mathrm{T}:=\hat{\bullet}(\mathrm{U})$ is a semistandard $(\mathscr{X}, \lambda)$-tableau.

(ii) If $u \nearrow v \in[\lambda]$ and $\mathrm{T}(u)=\mathrm{T}(v)$, then $\mathrm{U}(u) \prec_{\mathscr{X}} \bullet \mathrm{U}(v)$.

We say then that $\mathrm{U}$ is a $\bullet$-standardization of $\mathrm{T}$.

Example 3. Take $\mathscr{X}=\{\hat{1}<1<\hat{2}<2<\hat{3}<3\}$, with odd elements indicated by carets. Let $\lambda=(4,4,2)$. An $(\mathscr{X}, \lambda)$-tableau $\mathrm{T}$, its dual $\mathrm{T}^{*}$, conjugate $\mathrm{T}^{\prime}$, and dual conjugate $\mathrm{T}^{\prime *}$ are shown below.
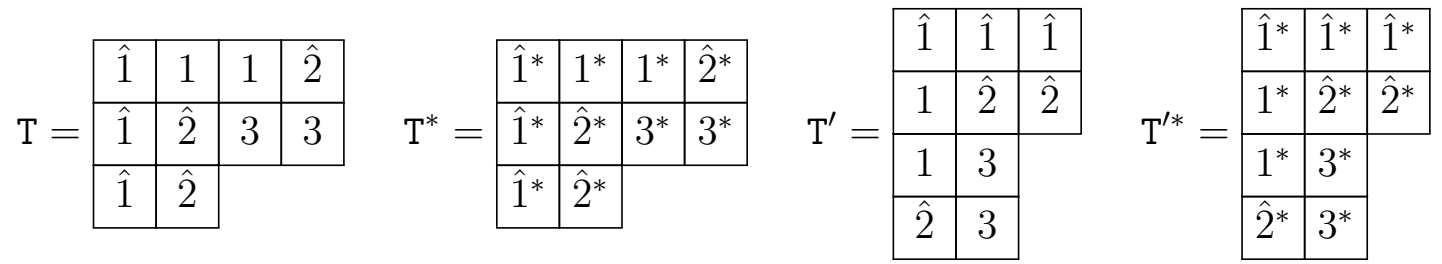
Then $\mathrm{T}$ is a semistandard $(\mathscr{X}, \lambda)$-tableau, and $\mathrm{T}^{*}$ is a semistandard $\left(\mathscr{X}^{*}, \lambda^{\prime}\right)$-tableau. In the standardizing alphabet $\mathscr{X}^{\bullet}$ we have

$$
\hat{1}^{(1)}<\hat{1}^{(2)}<\hat{1}^{(3)}<1^{(1)}<1^{(2)}<\hat{2}^{(1)}<\hat{2}^{(2)}<\hat{2}^{(3)}<3^{(1)}<3^{(2)},
$$

and

$$
\hat{2}^{(3)} \prec \hat{2}^{(2)} \prec \hat{2}^{(1)} \prec \hat{1}^{(3)} \prec \hat{1}^{(2)} \prec \hat{1}^{(1)} \prec 1^{(1)} \prec 1^{(2)} \prec 3^{(1)} \prec 3^{(2)},
$$

and so the $(\mathscr{X} \bullet, \lambda)$-tableau

$$
\mathrm{U}=\begin{array}{|l|l|l|l|}
\hat{1}^{(1)} & 1^{(1)} & 1^{(2)} & \hat{2}^{(1)} \\
\hline \hat{1}^{(2)} & \hat{2}^{(2)} & 3^{(1)} & 3^{(2)} \\
\hline \hat{1}^{(3)} & \hat{2}^{(3)} & & \\
\cline { 1 - 2 } &
\end{array}
$$

is a •-standardization of $\mathrm{T}$.

\section{$3 \quad$ Insertion and Extraction}

It will be convenient in practice to formally extend the domain and range of an $(\mathscr{X}, \lambda)$ tableau $\mathrm{T}$ to a function $\mathrm{T}: \mathrm{N} \rightarrow \mathscr{X} \cup\{\infty\}$ by setting $\mathrm{T}(u)=\infty$ for all $u \notin[\lambda]$. We extend the order $<$ on $\mathscr{X}$ to $\mathscr{X} \cup\{\infty\}$ by setting $x<\infty$ for all $x \in \mathscr{X}$. We define the symbols $\overline{0}:=<$ and $\underset{<}{\overline{1}}:=\leqslant$.

\subsection{Insertion}

Let $\lambda \vdash n$, and assume $\mathrm{T}$ is a semistandard $(\mathscr{X}, \lambda)$-tableau. Let $\varepsilon \in \mathbb{Z}_{2}$ and $x \in \mathscr{X}$. From this data we construct an $(\mathscr{X}, \mu)$-tableau $(\mathrm{T} \stackrel{\varepsilon}{\leftarrow} x)$, where $\mu \vdash n+1$, via the method of E-insertion.

\section{Algorithm for $\varepsilon$-insertion.}

(1) Set $i:=1, j:=1$, and $x_{1}:=x$.

(2) Set $b_{j}$ to be the smallest node in $\mathrm{N}\left(\varepsilon+\bar{x}_{j}, i\right)$ such that $x_{j} \stackrel{\varepsilon}{<} \mathrm{T}\left(b_{j}\right)$.

(3) If $\mathrm{T}\left(b_{j}\right)=\infty$, go to step (5). Otherwise, set $j:=j+1$.

(4) Set $x_{j}:=\mathrm{T}\left(b_{j-1}\right)$. Set $i:=\left(b_{j-1}\right)_{\varepsilon+\bar{x}_{j}}+1$ and go to step (2).

(5) Define $\mu$ such that $[\mu]:=[\lambda] \cup\left\{b_{j}\right\}$. Define $(\mathrm{T} \stackrel{\varepsilon}{\leftarrow} x)$ to be the $(\mathscr{X}, \mu)$-tableau such that $(\mathrm{T} \stackrel{\varepsilon}{\leftarrow} x)\left(b_{k}\right)=x_{k}$ for all $1 \leqslant k \leqslant j$, and $(\mathrm{T} \stackrel{\varepsilon}{\leftarrow} x)(u)=\mathrm{T}(u)$ for all other nodes of $[\mu]$. 
Assuming the process terminates when $j=m$, we call $b_{1}, \ldots, b_{m}$ the bumped node sequence, and call $A(\mathrm{~T}, \varepsilon, x):=b_{m}$ the added node. We call $x_{1}, \ldots, x_{m}$ the bumped letter sequence.

Remark 4. Informally speaking, under $\overline{0}$-insertion, bumped even letters are inserted in the next row down and bumped odd letters are inserted in the next column to the right. In


assure that semistandardness is maintained under $\varepsilon$-insertion, as will be shown in Lemma 12 .

Remark 5. If $\mathscr{X}=\mathbb{N}$, where $<$ is the usual order on integers and every element is of even parity, then $\overline{0}$-insertion is Schensted insertion [S]. For general $\mathscr{X}$ and standard tableaux, $\overline{0}$-insertion is mixed insertion as defined by Haiman (where odd letters are referred to as circled) $[\mathrm{H}]$.

Remark 6. In [SW, §3], Shimozono and White define a process called doubly-mixed insertion, also adapted from from $[\mathrm{H}]$, which is very similar to the $\varepsilon$-insertion presented in this paper; the processes are identical when applied to the subclass of standard tableaux. We note however that Shimozono and White use a different definition for semistandard tableaux - in their setup, semistandard tableaux are row-weak and column-strict with respect to both parities - so doubly-mixed insertion and $\varepsilon$-insertion differ substantially in the presence of repeated letters.

Example 7. With $\mathscr{X}$ as in Example 3 and $\mathrm{T}$ as shown below, we have
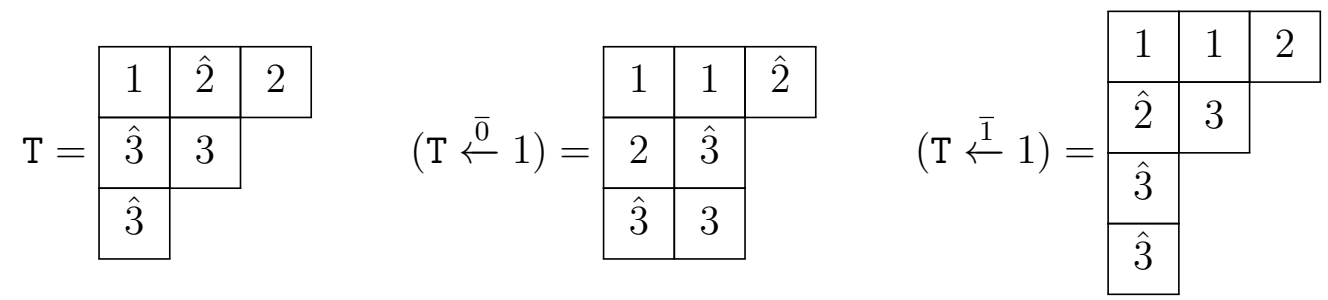

The bumped node sequence for $(\mathrm{T} \stackrel{\overline{0}}{\leftarrow} 1)$ is

$$
(1,2),(1,3),(2,1),(2,2),(3,2),
$$

and the bumped letter sequence is $1, \hat{2}, 2, \hat{3}, 3$. The bumped node sequence for $(\mathrm{T} \stackrel{\overline{1}}{\leftarrow} 1)$ is

$$
(1,1),(1,2),(2,1),(3,1),(4,1),
$$

and the bumped letter sequence is $1,1, \hat{2}, \hat{3}, \hat{3}$.

\subsection{Extraction}

Let $\mathrm{U}$ be a semistandard $(\mathscr{X}, \mu)$-tableau, let $u$ be a removable node of $[\mu]$, and set $\lambda$ to be such that $[\lambda]=[\mu] \backslash\{u\}$. We define a $(\mathscr{X}, \lambda)$-tableau $(\mathrm{U} \stackrel{\varepsilon}{\rightarrow} u)$ by the method of $\varepsilon$-extraction.

\section{Algorithm for $\varepsilon$-extraction.}


(1) Set $j:=1, c_{1}:=u$, and $y_{1}:=\mathrm{U}(u)$.

(2) Set $i:=\left(c_{j}\right)_{\varepsilon+\bar{y}_{j}}-1$. If $i=0$, go to step (5).

(3) Set $c_{j+1}$ to be the greatest node in $\mathrm{N}\left(\varepsilon+\bar{y}_{j}, i\right)$ such that $y_{j} \stackrel{\varepsilon}{>} \mathrm{U}\left(c_{j+1}\right)$.

(4) Set $j:=j+1$. Set $y_{j}:=\mathrm{U}\left(c_{j}\right)$. Go to step (2).

(5) Define $(\mathrm{U} \stackrel{\varepsilon}{\rightarrow} u)$ to be the $\lambda$-tableau such that $(\mathrm{U} \stackrel{\varepsilon}{\rightarrow} u)\left(c_{k}\right)=y_{k-1}$ for all $2 \leqslant k \leqslant j$, and $(\mathrm{U} \stackrel{\varepsilon}{\rightarrow} u)(v)=\mathrm{U}(v)$ for all other nodes of $[\lambda]$.

Assuming the process terminates when $j=m$, we call $y_{1}, \ldots, y_{m}$ the unbumped letter sequence., and define $R(\mathrm{U}, \varepsilon, u):=y_{m}$ to be the extracted letter. We call $c_{1}, \ldots, c_{m}$ the unbumped node sequence.

Example 8. With $\mathscr{X}$ as in Example 3 and $\mathrm{T}$ as shown below, we have

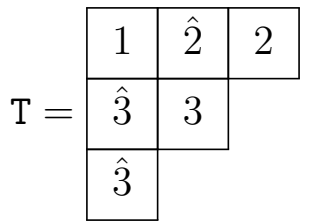

$$
(\mathrm{T} \stackrel{\overline{0}}{\rightarrow}(3,1))=\begin{array}{|l|l|l|}
\hline 1 & \hat{2} & 2 \\
\hline \hat{3} & 3 &
\end{array}
$$

$$
(\mathrm{T} \stackrel{\overline{1}}{\rightarrow}(3,1))=\begin{array}{|l|l|l|}
\hline 1 & 2 & \hat{3} \\
\hline \hat{3} & 3 & \multicolumn{1}{|c}{} \\
\cline { 1 - 2 } &
\end{array}
$$

The unbumped node sequence for $(\mathrm{T} \stackrel{\overline{0}}{\rightarrow}(3,1))$ is only the node $(3,1)$, and the unbumped letter sequence is $\hat{3}$.

The unbumped node sequence for $(\mathrm{T} \stackrel{\overline{1}}{\rightarrow}(3,1))$ is

$$
(3,1),(2,1),(1,3),(1,2)
$$

and the unbumped letter sequence is $\hat{3}, \hat{3}, 2, \hat{2}$.

\subsection{Some results on insertion and extraction}

As noted in Remark 5, $\varepsilon$-insertion is an adaptation of 'mixed insertion' defined by Haiman $[\mathrm{H}]$. Although Haiman works with standard tableaux, he remarks that his results may be extended to the semistandard case in a straightforward manner - this is outlined in $[\mathrm{H}, \S 1]$ and we take some pains to make the idea explicit in Lemma 14. Though some of the results in this subsection would follow from those in $[\mathrm{H}]$ and Lemma 14, we nevertheless include full proofs working in the general semistandard case, for the sake of clarity and self-containment, and since our notation and approach differ substantially from that of $[\mathrm{H}]$.

The following two lemmas follow directly from definitions of the algorithms. 
Lemma 9. Let $\mathrm{T}$ be a standard $(\mathscr{X}, \lambda)$-tableau, and assume $x \in \mathscr{X} \backslash \mathrm{T}$. Then

(i) $(\mathrm{T} \stackrel{\varepsilon}{\leftarrow} x)^{\prime}=\left(\mathrm{T}^{\prime} \stackrel{\varepsilon+\overline{1}}{\longleftarrow} x\right)$, and if $b_{1}, \ldots, b_{m}$ is the bumped node sequence for $(\mathrm{T} \stackrel{\varepsilon}{\leftarrow} x)$, then $b_{1}^{\prime}, \ldots, b_{m}^{\prime}$ is the bumped node sequence for $\left(\mathrm{T}^{\prime} \stackrel{\varepsilon+\overline{1}}{\longleftarrow} x\right)$.

(ii) $(\mathrm{T} \stackrel{\varepsilon}{\leftarrow} x)^{*}=\left(\mathrm{T}^{*} \stackrel{\varepsilon+\overline{1}}{\longleftarrow} x^{*}\right)$, and both insertions have the same bumped node sequence.

Lemma 10. Let $\mathrm{T}$ be a semistandard $(\mathscr{X}, \lambda)$-tableau. Then

(i) $(\mathrm{T} \stackrel{\varepsilon}{\leftarrow} x)^{\prime *}=\left(\mathrm{T}^{\prime *} \stackrel{\varepsilon}{\leftarrow} x^{*}\right)$ for every $x \in \mathscr{X}$, and if $b_{1}, \ldots, b_{m}$ is the bumped node sequence for $(\mathrm{T} \stackrel{\varepsilon}{\leftarrow} x)$, then $b_{1}^{\prime}, \ldots, b_{m}^{\prime}$ is the bumped node sequence for $\left(\mathrm{T}^{*} \stackrel{\varepsilon}{\leftarrow} x^{*}\right)$.

(ii) $(\mathrm{T} \stackrel{\varepsilon}{\rightarrow} u)^{\prime *}=\left(\mathrm{T}^{\prime *} \stackrel{\varepsilon}{\rightarrow} u^{\prime}\right)$ for every removable node $u \in[\lambda]$, and if $c_{1}, \ldots, c_{m}$ is the unbumped node sequence for $(\mathrm{T} \stackrel{\varepsilon}{\rightarrow} u)$, then $c_{1}^{\prime}, \ldots, c_{m}^{\prime}$ is the unbumped node sequence for $\left(\mathrm{T}^{* *} \stackrel{\varepsilon}{\rightarrow} u^{\prime}\right)$.

Lemma 11. Let $b_{1}, \ldots, b_{m}$ be the bumped node sequence, and let $x_{1}, \ldots, x_{m}$ be the bumped letter sequence for the $\varepsilon$-insertion $(\mathrm{T} \stackrel{\varepsilon}{\leftarrow} x)$. Assume $i<j$. Then:

(i) $x_{i}<x_{j}$

(ii) $\mathrm{T}\left(b_{i}\right) \stackrel{\varepsilon}{<} \mathrm{T}\left(b_{j}\right)$

(iii) $(\mathrm{T} \stackrel{\varepsilon}{\leftarrow} x)\left(b_{i}\right) \stackrel{\varepsilon}{<}(\mathrm{T} \stackrel{\varepsilon}{\leftarrow} x)\left(b_{j}\right)$

(iv) $b_{i} \ngtr b_{j}$.

Proof. (i)-(iii) are obvious. For (iv), note that if $\mathrm{T}$ is semistandard, then $b_{i} \geqslant b_{j}$ and (ii) would imply $\mathrm{T}\left(b_{i}\right)=\mathrm{T}\left(b_{i+1}\right)=\cdots=\mathrm{T}\left(b_{j}\right), \varepsilon=\overline{1}$, and either $b_{j} \downarrow b_{i}$ or $b_{j} \rightarrow b_{i}$. In the former case, semistandardness implies that $\overline{\mathrm{T}\left(b_{i}\right)}=\overline{1}$, hence by the $\overline{1}$-insertion algorithm, $\left(b_{i+a}\right)_{\overline{0}}=\left(b_{i}\right)_{\overline{0}}+a$ for $0 \leqslant a \leqslant j-i$, so $b_{j}$ is in a lower row than $b_{i}$, a contradiction. In the latter case, semistandardness implies that $\overline{\mathrm{T}\left(b_{i}\right)}=\overline{0}$, hence by the insertion algorithm, $\left(b_{i+a}\right)_{\overline{1}}=\left(b_{i}\right)_{\overline{1}}+a$ for $0 \leqslant a \leqslant j-i$, so $b_{j}$ is in a column rightward of $b_{i}$, a contradiction.

Lemma 12. Let $\mathrm{T}$ be a semistandard $(\mathscr{X}, \lambda)$-tableau.

(i) $(\mathrm{T} \stackrel{\varepsilon}{\leftarrow} x)$ is semistandard for every $x \in \mathscr{X}$.

(ii) $(\mathrm{T} \stackrel{\varepsilon}{\rightarrow} u)$ is semistandard for every removable node $u \in[\lambda]$.

Proof. (i) Let $b_{1}, \ldots, b_{m}$ be the bumped node sequence, and let $x_{1}, \ldots, x_{m}$ be the bumped letter sequence for the insertion $(\mathrm{T} \leftarrow \mathcal{\varepsilon} x)$. For $1 \leqslant i \leqslant m$, let $\mathrm{T}_{i}$ be the tableau defined by setting $\mathrm{T}_{i}\left(b_{j}\right)=x_{j}$ for $1 \leqslant j \leqslant i$, and $\mathrm{T}_{i}(u)=\mathrm{T}(u)$ for all other $u \in[\lambda]$. It is easy to see that $\mathrm{T}_{1}$ is semistandard. Now we argue that $\mathrm{T}_{k}$ is semistandard by induction. 
Assume $\varepsilon+\bar{x}_{k}=\overline{0}$. Then $\left(b_{k}\right)_{\overline{0}}=\left(b_{k-1}\right)_{\overline{0}}+1$ by the algorithm. If $z$ is the node directly below $b_{k-1}$, then $z \neq b_{i}$ for any $1 \leqslant i \leqslant k-1$ by Lemma 11(iv). By semistandardness of $\mathrm{T}_{k-1}$ then

$$
x_{k}=\mathrm{T}_{k-1}\left(b_{k-1}\right) \leqslant \mathrm{T}_{k-1}(z)=\mathrm{T}(z),
$$

so $b_{k} \rightarrow z$, and thus $b_{k} \nearrow b_{k-1}$. Let $l, r$ be the nodes to the immediate left and right of $b_{k}$, respectively. Then

$$
\mathrm{T}_{k}(l)=\mathrm{T}_{k-1}(l) \leqslant \mathrm{T}(l) \stackrel{\varepsilon+\overline{1}}{<} x_{k} \underset{<}{\varepsilon} \mathrm{T}\left(b_{k}\right)=\mathrm{T}_{k-1}\left(b_{k}\right) \leqslant \mathrm{T}_{k-1}(r)=\mathrm{T}_{k}(r) .
$$

Thus the $\left(b_{k}\right)_{0}$ th row of $\mathrm{T}_{k}$ is non-decreasing. Moreover, if $\bar{x}_{k}=\overline{1}$, then $\varepsilon=\overline{1}$, so $\mathrm{T}_{k}(l)<x_{k}=\mathrm{T}_{k}\left(b_{k}\right)$. If $x_{k}=\mathrm{T}_{k}(r)$, then $\mathrm{T}_{k-1}\left(b_{k}\right)=\mathrm{T}_{k-1}(r)$, yet both are odd, a contradiction of the semistandardness of $\mathrm{T}_{k-1}$. Thus $\mathrm{T}_{k}\left(b_{k}\right)=x_{k}<\mathrm{T}_{k}(r)$. Thus the $\left(b_{k}\right)_{0}$ th row of $\mathrm{T}_{k}$ is row-strict with respect to odd letters.

Let $u, d$ be the nodes directly above and below $b_{k}$, respectively. Then $u \rightarrow b_{k-1}$ or $u=b_{k-1}$. Then

$$
\mathrm{T}_{k}(u)=\mathrm{T}_{k-1}(u) \leqslant \mathrm{T}_{k-1}\left(b_{k-1}\right)=x_{k-1} \stackrel{\varepsilon}{<} x_{k} \stackrel{\varepsilon}{<} \mathrm{T}\left(b_{k}\right)=\mathrm{T}_{k-1}\left(b_{k}\right) \leqslant \mathrm{T}_{k-1}(d)=\mathrm{T}_{k}(d) .
$$

Thus the $\left(b_{k}\right)_{\overline{1}}$ th column of $\mathrm{T}_{k}$ is non-decreasing. Moreover, if $\bar{x}_{k}=\overline{0}$, then $\varepsilon=\overline{0}$, and $\mathrm{T}_{k}(u)<x_{k}=\mathrm{T}_{k}\left(b_{k}\right)<\mathrm{T}_{k}(d)$, so the $\left(b_{k}\right)_{1}$ th column of $\mathrm{T}_{k}$ is column-strict with respect to even letters.

Thus $\mathrm{T}_{k}$ is semistandard if $\varepsilon+\bar{x}_{k}=\overline{0}$. Assume on the other hand that $\varepsilon+\bar{x}_{k}=\overline{0}$. Let $\mathrm{U}=\mathrm{T}^{*}$. Then, applying the above argument to the insertion $\left(\mathrm{U} \stackrel{\varepsilon}{\leftarrow} x^{*}\right)$, we have that $\mathrm{U}_{k}$ is semistandard. But by Lemma $9, \mathrm{U}_{k}=\mathrm{T}_{k}^{* *}$. Thus by Lemma $2, \mathrm{~T}_{k}$ is semistandard. This completes the proof of (i).

(ii) Let $c_{1}, \ldots, c_{m}$ be the unbumped node sequence, and $y_{1}, \ldots, y_{m}$ be the unbumped letter sequence for the extraction $(\mathrm{T} \stackrel{\varepsilon}{\rightarrow} u$ ). Let $\mu$ be defined such that $[\mu]=[\lambda] \backslash\{u\}$. Let $\mathrm{T}_{1}$ be the $(\mathscr{X}, \mu)$-tableau defined by $\mathrm{T}_{1}(v)=\mathrm{T}(v)$ for all $v \in[\mu]$. For $2 \leqslant i \leqslant m$, let $\mathrm{T}_{i}$ be the $(\mathscr{X}, \mu)$-tableau defined by $\mathrm{T}_{i}\left(c_{j}\right)=y_{j-1}$ for $2 \leqslant j \leqslant i$, and $\mathrm{T}_{i}(v)=\mathrm{T}(v)$ otherwise. We have that $\mathrm{T}_{1}$ is semistandard by assumption. We show by induction that $\mathrm{T}_{k}$ is semistandard for all $k$.

Assume $\varepsilon+\bar{y}_{k-1}=\overline{0}$. Let $l, r$ be the nodes to the immediate left and right of $c_{k}$. Then

$$
\mathrm{T}_{k}(l)=\mathrm{T}_{k-1}(l) \leqslant \mathrm{T}_{k-1}\left(c_{k}\right)=\mathrm{T}\left(c_{k}\right) \stackrel{\varepsilon}{<} y_{k-1} \underset{<}{\varepsilon+\overline{1}} \mathrm{~T}(r)=\mathrm{T}_{k}(r) .
$$

Thus the $\left(c_{k}\right)_{\overline{0}}$-th row of $\mathrm{T}_{k}$ is non-decreasing. Moreover, if $\bar{y}_{k-1}=\overline{1}$, then $\varepsilon=\overline{1}$, so $\mathrm{T}_{k}\left(c_{k}\right)=y_{k-1}<\mathrm{T}_{k}(r)$. If $\mathrm{T}_{k}(l)=y_{k-1}$, then $\mathrm{T}_{k-1}(l)=\mathrm{T}_{k-1}\left(c_{k}\right)$, yet both are odd, a contradiction since $\mathrm{T}_{k-1}$ is semistandard by assumption. Thus $\mathrm{T}_{k}$ is row-strict with respect to odd letters.

Let $u, d$ be the nodes directly above and below $c_{k}$, respectively. Then $c_{k-1} \nearrow c_{k}$ and $c_{k}$ is in the row above $c_{k-1}$. So $c_{k-1} \rightarrow d$ or $c_{k-1}=d$. In either case we have

$$
\mathrm{T}_{k}(u)=\mathrm{T}_{k-1}(u) \leqslant \mathrm{T}_{k-1}\left(c_{k}\right)=\mathrm{T}_{k}\left(c_{k}\right) \stackrel{\varepsilon}{<} y_{k-1} \stackrel{\varepsilon}{<} y_{k-2}=\mathrm{T}_{k-1}\left(c_{k-1}\right) \leqslant \mathrm{T}_{k-1}(d)=\mathrm{T}_{k}(d) .
$$


Thus the $\left(c_{k}\right)_{\overline{1}}$-th column of $\mathrm{T}_{k}$ is non-decreasing. Moreover, if $\bar{y}_{k-1}=\overline{0}$, then $\varepsilon=\overline{0}$, so $\mathrm{T}_{k}(u)<y_{k-1}=\mathrm{T}_{k}\left(c_{k}\right)<\mathrm{T}_{k}(d)$, and thus $\mathrm{T}_{k}$ is column-strict with respect to even letters.

Therefore $\mathrm{T}_{k}$ is semistandard if $\varepsilon+\bar{y}_{k-1}=\overline{0}$. On the other hand assume $\varepsilon+\bar{y}_{k-1}=\overline{1}$. Let $\mathrm{U}=\mathrm{T}^{\prime *}$. Then, applying the above argument to the extraction $\left(\mathrm{U} \stackrel{\varepsilon}{\rightarrow} u^{\prime}\right)$, we have that $\mathrm{U}_{k}$ is semistandard. But $\mathrm{U}_{k}=\mathrm{T}_{k}^{* *}$ by Lemma 9. Thus by Lemma 2, $\mathrm{T}_{k}$ is semistandard.

Lemma 13. Let $\mathrm{T}$ be a semistandard $(\mathscr{X}, \lambda)$-tableau.

(i) $\mathrm{T}=((\mathrm{T} \stackrel{\varepsilon}{\leftarrow} x) \stackrel{\varepsilon}{\rightarrow} A(\mathrm{~T}, \varepsilon, x))$ for every $x \in \mathscr{X}$.

(ii) $\mathrm{T}=((\mathrm{T} \stackrel{\varepsilon}{\rightarrow} u) \stackrel{\varepsilon}{\leftarrow} R(\mathrm{~T}, \varepsilon, u))$ for every removable node $u \in[\lambda]$.

Proof. By Lemma 12, $(\mathrm{T} \stackrel{\varepsilon}{\leftarrow} x)$ and $(\mathrm{T} \stackrel{\varepsilon}{\rightarrow} u)$ are semistandard tableaux, and $\varepsilon$-insertion and $\varepsilon$-extraction are inverse processes by construction.

The following lemma is a key tool in generalizing some results proved for standard tableaux to the more general case of semistandard tableaux.

Lemma 14. Let $\mathrm{T}$ be a semistandard $(\mathscr{X}, \lambda)$-tableau, and let $\mathrm{T}^{\bullet}$ be a $\bullet$-standardization of T. Let $y \in \mathscr{X}^{\bullet}$ be such that

(i) $x \prec y$ if $\varepsilon+\bar{y}=\overline{0}$,

(ii) $x \succ y$ if $\varepsilon+\bar{y}=\overline{1}$,

for every $x \in \mathrm{T}^{\bullet}$ such that $\hat{\bullet}(x)=\hat{\bullet}(y)$. Then $\left(\mathrm{T}^{\bullet} \stackrel{\varepsilon}{\leftarrow} y\right)$ is a $\bullet$-standardization of $(\mathrm{T} \stackrel{\varepsilon}{\leftarrow}$ $\hat{\boldsymbol{\bullet}}(y))$. Moreover, if $b_{1}, \ldots, b_{k}$ is the bumped node sequence for the insertion $(\mathrm{T} \stackrel{\varepsilon}{\varepsilon} \hat{\bullet}(y))$, and $b_{1}^{\bullet}, \ldots, b_{m}^{\bullet}$ is the bumped node sequence for the insertion $\left(\mathrm{T}^{\bullet} \stackrel{\varepsilon}{\leftarrow} y\right)$, then $k=m$ and $b_{i}=b_{i}^{\bullet}$ for all $i$.

Proof. We will first prove the result in the case $\varepsilon=\overline{0}$, so that $x<y$ for all $x \in \mathrm{T}^{\bullet}$ such that $\hat{\bullet}(x)=\hat{\bullet}(y)$. We will write $\mathrm{T}_{y}^{\bullet}$ for $\left(\mathrm{T}^{\bullet} \stackrel{\overline{0}}{\leftarrow} y\right)$ and $\mathrm{T} \hat{\bullet}(y)$ for $(\mathrm{T} \stackrel{\overline{0}}{\leftarrow} \hat{\bullet}(y))$. We will also write $x_{1}, \ldots, x_{k}$ be the bumped letter sequence for the insertion $(\mathrm{T} \leftarrow \dot{\varepsilon} \hat{\bullet}(y)$ ), and $x_{1}^{\bullet}, \ldots, x_{m}^{\bullet}$ for the bumped letter sequence for the insertion $\left(\mathrm{T}^{\bullet} \stackrel{\varepsilon}{\leftarrow} y\right)$. We will first prove by induction that $b_{i}=b_{i}^{\bullet}$ for all $i$, hence $k=m$.

We have $y<\mathrm{T}^{\bullet}\left(b_{1}^{\bullet}\right)$, so $\hat{\bullet}(y) \leqslant \hat{\bullet}\left(\mathrm{T}^{\bullet}\left(b_{1}^{\bullet}\right)\right)=\mathrm{T}\left(b_{1}^{\bullet}\right)$. Moreover, by the assumption on $y$, we have that $\hat{\bullet}(y) \neq \mathrm{T}\left(b_{1}^{\bullet}\right)$, so $\hat{\bullet}(y)<\mathrm{T}\left(b_{1}^{\bullet}\right)$. If $\bar{y}=\overline{0}$ (resp. if $\bar{y}=\overline{1}$ ), let $u$ be the node directly to the left (resp. directly above) of $b_{1}^{\bullet}$. Then $\mathrm{T}^{\bullet}(u)<y$, so $\mathrm{T}(u) \leqslant \hat{\bullet}(y)$. Thus $b_{1}=b_{1}^{\bullet}$.

Now assume that $b_{i}=b_{i}^{\bullet}$. If $\overline{\mathrm{T}^{\bullet}\left(b_{i}^{\bullet}\right)}=\overline{0}$ (resp. if $\overline{\mathrm{T}^{\bullet}\left(b_{i}^{\bullet}\right)}=\overline{1}$ ), then $b_{i+1}^{\bullet} \nearrow b_{i}$ (resp. $\left.b_{i} \nearrow b_{i+1}^{\bullet}\right)$, so $\mathrm{T}^{\bullet}\left(b_{i+1}^{\bullet}\right)<\mathrm{T}^{\bullet}\left(b_{i}\right)$ if $\mathrm{T}\left(b_{i+1}^{\bullet}\right)=\mathrm{T}\left(b_{i}\right)$, since $\mathrm{T}^{\bullet}$ is a $\bullet$-standardization of $\mathrm{T}$. However, we also have $\mathrm{T}^{\bullet}\left(b_{i}\right)<\mathrm{T}^{\bullet}\left(b_{i+1}^{\bullet}\right)$, so $\mathrm{T}\left(b_{i}\right) \leqslant \mathrm{T}\left(b_{i+1}^{\bullet}\right)$, and hence $x_{i+1}=\mathrm{T}\left(b_{i}\right)<$ 
$\mathrm{T}\left(b_{i+1}^{\bullet}\right)$. If $\overline{\mathrm{T}^{\bullet}\left(b_{i}^{\bullet}\right)}=\overline{0}$ (resp. if $\overline{\mathrm{T}^{\bullet}\left(b_{i}^{\bullet}\right)}=\overline{1}$ ), let $u$ be the node directly to the left (resp. directly above) $b_{i+1}^{\bullet}$. Then $\mathrm{T}^{\bullet}(u)<\mathrm{T}^{\bullet}\left(b_{i}\right)$, so $\mathrm{T}(u) \leqslant \mathrm{T}\left(b_{i}\right)=x_{i+1}$, and thus $b_{i+1}=b_{i+1}^{\bullet}$.

Therefore we have that $\hat{\boldsymbol{\theta}}\left(\mathrm{T}_{y}^{\bullet}\right)=\mathrm{T}_{\hat{\boldsymbol{\bullet}}(y)}$, and by construction $\mathrm{T}_{y}^{\bullet}$ is standard. Now we show that $\mathrm{T}_{y}^{\bullet}$ is $\bullet$-standard. Let $u \nearrow v \in\left[\operatorname{sh}\left(\mathrm{T}_{y}^{\bullet}\right)\right]$, and assume $\mathrm{T}_{\hat{\boldsymbol{o}}(y)}(u)=\mathrm{T}_{\hat{\boldsymbol{o}}(y)}(v)$. If neither $u$ nor $v$ is equal to a bumped node $b_{i}^{\bullet}$, then the result follows since $\mathrm{T}^{\bullet}$ is $\bullet$-standard. On the other hand if both are bumped nodes, say $u=b_{i}$ and $v=b_{j}$, then $\mathrm{T}_{y}^{\bullet}(u)=x_{i}^{\bullet}, \mathrm{T}_{y}^{\bullet}(v)=x_{j}^{\bullet}$, and $x_{i}=x_{j}$. But this cannot happen in $\overline{0}$-insertion for distinct $i$ and $j$. This leaves the cases where exactly one of $u, v$ is a bumped node. We consider the two cases separately:

(a) Assume that $v=b_{i}$ is a bumped node, and $u$ is not. Then $\mathrm{T}_{y}^{\bullet}(v)=x_{i}^{\bullet}$, and $\mathrm{T}(u)=\mathrm{T}_{\hat{\boldsymbol{o}}(y)}(u)=\mathrm{T}_{\hat{\boldsymbol{o}}(y)}(v)=x_{i}$. Note that if $\bar{y}=\overline{1}$, then $\overline{\boldsymbol{\bullet}(y)}=\overline{1}$, and $b_{1}$ is in the first column. Then by Lemma 11, $\hat{\bullet}(y)=\mathrm{T}_{\hat{\boldsymbol{\bullet}}(y)}\left(b_{1}\right)<\mathrm{T}_{\hat{\boldsymbol{o}}(y)}(w)$ for every node $w$ directly below $b_{1}$. Then, since $\mathrm{T}_{\hat{\boldsymbol{\bullet}}(y)}$ is semistandard, $\mathrm{T}_{\hat{\boldsymbol{o}}(y)}\left(b_{1}\right)<\mathrm{T}_{\hat{\boldsymbol{\bullet}}(y)}(w)$ for every node $w$ such that $b_{1} \searrow w$. Thus $b_{1} \nearrow w$ for every node $w$ such that $\hat{\bullet}(y)=\mathrm{T}_{\hat{\boldsymbol{\bullet}}(y)}\left(b_{1}\right)=\mathrm{T}_{\hat{\boldsymbol{}}(y)}(w)$. Thus if $i=1$, then $\bar{y}=\overline{0}$ and by the assumption on $y$, $\mathrm{T}_{y}^{\bullet}(u)=\mathrm{T}^{\bullet}(u)<y=x_{1}^{\bullet}=\mathrm{T}_{y}^{\bullet}(v)$ as required.

Assume $i \geqslant 2$. Then $x_{i}^{\bullet}=\mathrm{T}^{\bullet}\left(b_{i-1}\right)$. If $\bar{x}_{i}=\overline{0}$, then $b_{i} \nearrow b_{i-1}$. Thus $u \nearrow b_{i-1}$, so $\mathrm{T}_{y}^{\bullet}(u)=\mathrm{T}^{\bullet}(u)<\mathrm{T}^{\bullet}\left(b_{i-1}\right)=\mathrm{T}_{y}^{\bullet}(v)$ since $\mathrm{T}^{\bullet}$ is $\hat{\bullet}$-standard. Assume $\bar{x}_{i}=\overline{1}$. Then $b_{i-1} \nearrow b_{i}=v$. If $v$ is directly above $u$, then $\mathrm{T}_{y}^{\bullet}(v)<\mathrm{T}_{y}^{\bullet}(u)$ since $\mathrm{T}_{y}^{\bullet}$ is standard. Assume $v$ is not directly above $u$. If it is not the case that $u \nearrow b_{i-1}$, then it must be that $u \searrow b_{i-1}$. But then since $\mathrm{T}_{y}\left(b_{i-1}\right)=x_{i-1}<\mathrm{T}\left(b_{i-1}\right)=\mathrm{T}(u)=\mathrm{T}_{y}(u)$, this cannot be true. Therefore $u \nearrow b_{i-1}$, and again we have $\mathrm{T}_{y}^{\bullet}(u)=\mathrm{T}^{\bullet}(u)>\mathrm{T}^{\bullet}\left(b_{i-1}\right)=\mathrm{T}_{y}(v)$, as required.

(b) Assume that $u=b_{i}$ is a bumped node, and $v$ is not. Then $\mathrm{T}_{y}^{\bullet}(u)=x_{i}^{\bullet}$, and $\mathrm{T}(v)=\mathrm{T}_{\hat{\boldsymbol{o}}(y)}(v)=\mathrm{T}_{\hat{\boldsymbol{o}}(y)}(u)=x_{i}$. Note that if $\bar{y}=\overline{0}$, then $w \nearrow b_{1}$ for every node $w$ such that $\hat{\bullet}(y)=\mathrm{T}_{\hat{\boldsymbol{\bullet}}(y)}\left(b_{1}\right)=\mathrm{T}_{\hat{\boldsymbol{\bullet}}(y)}(w)$. Thus if $i=1$, then $\bar{y}=\overline{1}$ and $\mathrm{T}^{\bullet}(u)=y=x_{1}^{\bullet}>\mathrm{T}^{\bullet}(v)$ as required.

Assume $i \geqslant 2$. Then $x_{i}^{\bullet}=\mathrm{T}^{\bullet}\left(b_{i-1}\right)$. If $\bar{x}_{i}=\overline{1}$, then $b_{i-1} \nearrow b_{i}$. Thus $b_{i-1} \nearrow v$, so $\mathrm{T}_{y}^{\bullet}(u)=\mathrm{T}^{\bullet}\left(b_{i-1}\right)>\mathrm{T}^{\bullet}(v)=\mathrm{T}_{y}^{\bullet}(v)$. Assume $\bar{x}_{i}=\overline{0}$. If $v$ is directly to the right of $u$, then $\mathrm{T}_{y}^{\bullet}(u)<\mathrm{T}_{y}^{\bullet}(v)$. Assume $v$ is not directly to the right of $u$. Then if it is not the case that $b_{i-1} \nearrow v$, then it must be that $v \searrow b_{i-1}$. But then since $\mathrm{T}_{y}\left(b_{i-1}\right)=x_{i-1}<\mathrm{T}\left(b_{i-1}\right)=\mathrm{T}(v)=\mathrm{T}_{y}(v)$, this cannot be true. Therefore $b_{i-1} \nearrow v$, and thus we have $\mathrm{T}_{y}^{\bullet}(u)=\mathrm{T}^{\bullet}\left(b_{i-1}\right)>\mathrm{T}^{\bullet}(v)=\mathrm{T}_{y}(v)$, as required.

This completes the proof of the lemma when $\varepsilon=\overline{0}$. Now assume $\varepsilon=\overline{1}$. Then $x>y$ for all $x \in \mathrm{T}^{\bullet}$. This proof proceeds along the same lines as the first part, but because there is an inherent discrepancy in the comparisons $\stackrel{\overline{0}}{<}=<$ and $\underset{<}{\overline{1}}=\leqslant$ we will provide the details in full. We'll write $T_{y}^{\bullet}$ for $\left(T^{\bullet} \stackrel{\overline{1}}{\leftarrow} y\right)$ and $\mathrm{T}_{\hat{\bullet}(y)}$ for $(\mathrm{T} \stackrel{\overline{1}}{\leftarrow} \hat{\bullet}(y))$. We will prove by induction that $b_{i}=b_{i}^{\bullet}$ for all $i$, hence $k=m$.

We have $y<\mathrm{T}^{\bullet}\left(b_{1}^{\bullet}\right)$, so $\hat{\bullet}(y) \leqslant \hat{\bullet}\left(\mathrm{T}^{\bullet}\left(b_{1}^{\bullet}\right)\right)=\mathrm{T}\left(b_{1}^{\bullet}\right)$ since $\mathrm{T}^{\bullet}$ is a $\bullet$-standardization of $\mathrm{T}$. If $\bar{y}=\overline{0}$ (resp. if $\bar{y}=\overline{1}$ ), let $u$ be the node directly above (resp. directly to the left of) 
$b_{1}^{\bullet}$. Then $\mathrm{T}^{\bullet}(u)<y$, so $\mathrm{T}(u) \leqslant \hat{\bullet}(y)$. Moreover, by the assumption on $y$, we have that $\hat{\bullet}(y) \neq \mathrm{T}(u)$, so $\hat{\bullet}(y)>\mathrm{T}(u)$. Thus $b_{1}=b_{1}^{\bullet}$.

Now assume that $b_{i}=b_{i}^{\bullet}$. We have $x_{i+1}^{\bullet}=\mathrm{T}^{\bullet}\left(b_{i}\right)<\mathrm{T}^{\bullet}\left(b_{i+1}^{\bullet}\right)$, so $x_{i+1}=\mathrm{T}\left(b_{i}\right) \leqslant \mathrm{T}\left(b_{i+1}^{\bullet}\right)$. If $\overline{\mathrm{T}^{\bullet}\left(b_{i}\right)}=\overline{1}$ (resp. $\overline{\mathrm{T}^{\bullet}\left(b_{i}\right)}=\overline{0}$ ), let $u$ be the node directly to the left of (resp. directly above) $b_{i+1}^{\bullet}$, and note that $u \nearrow b_{i}$ (resp. $b_{i} \nearrow u$ ), so that $\mathrm{T}^{\bullet}(u)>\mathrm{T}^{\bullet}\left(b_{i}\right)=x_{i+1}^{\bullet}$ if $\mathrm{T}(u)=\mathrm{T}\left(b_{i}\right)=x_{i+1}$. But $x_{i+1}^{\bullet}>\mathrm{T}^{\bullet}(u)$ by the definition of $b_{i+1}^{\bullet}$, so it must be that $\mathrm{T}(u) \neq x_{i+1}$. Therefore $x_{i+1}>\mathrm{T}(u)$, and so $b_{i+1}=b_{i+1}^{\bullet}$.

Therefore we have that $\hat{\bullet}\left(T_{y}^{\bullet}\right)=T_{\hat{\bullet}(y)}$, and by construction $\mathrm{T}_{y}^{\bullet}$ is standard. Now we show that $\mathrm{T}_{y}^{\bullet}$ is $\bullet$-standard. Let $u \nearrow v \in\left[\operatorname{sh}\left(\mathrm{T}_{y}^{\bullet}\right)\right]$, and assume $\mathrm{T}_{\boldsymbol{\bullet}(y)}(u)=\mathrm{T}_{\boldsymbol{\bullet}(y)}(v)$. If neither $u$ nor $v$ is equal to a bumped node $b_{i}^{\bullet}$, then the result follows since $\mathrm{T}^{\bullet}$ is $\bullet$-standard.

On the other hand if both are bumped nodes, say $u=b_{i}$ and $v=b_{j}$, then $\mathrm{T}_{y}^{\bullet}(u)=x_{i}^{\bullet}$, $\mathrm{T}_{y}^{\bullet}(v)=x_{j}^{\bullet}$, and $x_{i}=x_{j}$. Note in general that if $x_{k}=x_{k+1}$, then $b_{k} \nearrow b_{k+1}$ if $\bar{x}_{k}=\overline{0}$, and $b_{k+1} \nearrow b_{k}$ if $\bar{x}_{k}=\overline{1}$. Thus, if $i<j$, then $x_{i}=x_{i+1}=\cdots=x_{j}$ and $b_{i} \nearrow b_{j}$ imply that $\bar{x}_{i}=\overline{0}$ and $\mathrm{T}_{y}^{\bullet}(u)=\mathrm{T}_{y}^{\bullet}\left(b_{i}\right)=x_{i}^{\bullet}<x_{j}^{\bullet}=\mathrm{T}_{y}^{\bullet}\left(b_{j}\right)=\mathrm{T}_{y}^{\bullet}(v)$, as required. On the other hand, if $j<i$, then $x_{j}=x_{j+1}=\cdots=x_{i}$ and $b_{i} \nearrow b_{j}$ imply that $\bar{x}_{i}=\overline{1}$ and $\mathrm{T}_{y}^{\bullet}(u)=\mathrm{T}_{y}^{\bullet}\left(b_{i}\right)=x_{i}^{\bullet}>x_{j}^{\bullet}=\mathrm{T}_{y}^{\bullet}\left(b_{j}\right)=\mathrm{T}_{y}^{\bullet}(v)$, as required. This leaves the cases where exactly one of $u, v$ is a bumped node. We consider the two cases separately:

(a) Assume that $v=b_{i}$ is a bumped node, and $u$ is not. Then $\mathrm{T}_{y}^{\bullet}(v)=x_{i}^{\bullet}$, and $\mathrm{T}_{\hat{\boldsymbol{\bullet}}(y)}(u)=\mathrm{T}_{\hat{\boldsymbol{\bullet}}(y)}(v)=x_{i}$. Note that if $\bar{y}=\overline{0}$, then $b_{1} \nearrow w$ for every node $w$ such that $\hat{\bullet}(y)=\mathrm{T}_{\hat{\bullet}(y)}(w)$. Thus if $i=1$, then $\bar{y}=\overline{1}$ and $\mathrm{T}_{y}^{\bullet}(u)=\mathrm{T}^{\bullet}(u)>y=x_{1}^{\bullet}=\mathrm{T}_{y}^{\bullet}(v)$ as required.

Assume $i \geqslant 2$. Then $x_{i}^{\bullet}=\mathrm{T}^{\bullet}\left(b_{i-1}\right)$. If $\bar{x}_{i}=\overline{1}$, then $b_{i} \nearrow b_{i-1}$. Thus $u \nearrow b_{i-1}$, so $\mathrm{T}_{y}^{\bullet}(u)=\mathrm{T}^{\bullet}(u)>\mathrm{T}^{\bullet}\left(b_{i-1}\right)=\mathrm{T}_{y}^{\bullet}(v)$. Assume $\bar{x}_{i}=\overline{0}$. Since $\mathrm{T}_{\hat{\bullet}(y)}(u)=\mathrm{T}_{\hat{\bullet}(y)}(v)=x_{i}$ and $\mathrm{T}_{\hat{\boldsymbol{\bullet}}(y)}$ is semistandard, it cannot be that $v$ is directly above $u$. If it is not the case that $u \nearrow b_{i-1}$, then it must be that $u \searrow b_{i-1}$. Moreover since $\mathrm{T}(u)=\mathrm{T}\left(b_{i-1}\right)$ and $\mathrm{T}$ is semistandard, it cannot be that $u$ is directly above $b_{i-1}$, so $u \Downarrow b_{i-1}$. But then $\mathrm{T}_{\hat{\bullet}(y)}(u)=\mathrm{T}(u)<\mathrm{T}\left(b_{i-1}\right)=\mathrm{T}_{\boldsymbol{\bullet}(y)}(v)$, a contradiction. Therefore $u \nearrow b_{i-1}$, and thus we have $\mathrm{T}_{y}^{\bullet}(u)=\mathrm{T}^{\bullet}(u)<\mathrm{T}^{\bullet}\left(b_{i-1}\right)=\mathrm{T}_{y}^{\bullet}(v)$, as required.

(b) Assume that $u=b_{i}$ is a bumped node, and $v$ is not. Then $\mathrm{T}^{\bullet}(u)=x_{i}^{\bullet}$, and $\mathrm{T}_{\hat{\boldsymbol{\bullet}}(y)}(u)=\mathrm{T}_{\hat{\boldsymbol{\bullet}}(y)}(v)=\mathrm{T}(v)=x_{i}$. Note that if $\bar{y}=\overline{1}$, then $w \nearrow b_{1}$ for every node $w$ such that $\hat{\bullet}(y)=\mathrm{T}_{\boldsymbol{\bullet}(y)}(w)$. Thus if $i=1$, then $\bar{y}=\overline{0}$ and $\mathrm{T}^{\bullet}(u)=y=x_{1}^{\bullet}<\mathrm{T}^{\bullet}(v)$ as required.

Assume $i \geqslant 2$. Then $x_{i}^{\bullet}=\mathrm{T}^{\bullet}\left(b_{i-1}\right)$. If $\bar{x}_{i}=\overline{0}$, then $b_{i-1} \nearrow b_{i}$. Thus $b_{i-1} \nearrow v$, so $\mathrm{T}_{y}^{\bullet}(u)=\mathrm{T}^{\bullet}\left(b_{i-1}\right)<\mathrm{T}^{\bullet}(v)=\mathrm{T}_{y}^{\bullet}(v)$. Assume $\bar{x}_{i}=\overline{1}$. Since $\mathrm{T}_{\hat{\boldsymbol{\bullet}}(y)}(u)=\mathrm{T}_{\hat{\boldsymbol{}}(y)}(v)=x_{i}$ and $\mathrm{T}_{\hat{\boldsymbol{}}(y)}$ is semistandard, it cannot be that $v$ is directly to the right of $u$. If it is not the case that $b_{i-1} \nearrow v$, then it must be that $v \searrow b_{i-1}$. Moreover since $\mathrm{T}(v)=\mathrm{T}\left(b_{i-1}\right)$ and $\mathrm{T}$ is semistandard, it cannot be that $b_{i-1}$ is directly to the right of $v$, so $v \Downarrow b_{i-1}$. But then $\mathrm{T}_{\hat{\bullet}(y)}(u)=\mathrm{T}\left(b_{i-1}\right)>\mathrm{T}(v)=\mathrm{T}_{\hat{\bullet}(y)}(v)$, a contradiction. Therefore $b_{i-1} \nearrow v$, and thus we have $\mathrm{T}_{y}^{\bullet}(u)=\mathrm{T}^{\bullet}\left(b_{i-1}\right)>\mathrm{T}^{\bullet}(v)=\mathrm{T}_{y}^{\bullet}(v)$, as required. 
This completes the proof of the lemma in the case $\varepsilon=\overline{1}$.

\section{Behavior of bumped nodes}

In this section we prove some technical results on the distribution of bumped nodes in $\varepsilon$-insertion. These results generalize some of those in $[\mathrm{K}]$ to the superalphabet setting, and are key in proving Theorem 21. Moreover, as bounds on the distribution of bumped nodes in successive insertions are examined in this section, the results may be of independent interest in the area of tableaux growth.

\subsection{Bumped node distribution}

Lemma 15. Let $\mathrm{T}$ be a semistandard $(\mathscr{X}, \lambda)$-tableau, $\varepsilon, \delta \in \mathbb{Z}_{2}$, and $x \in \mathscr{X}$. Let $b_{1}, \ldots, b_{m}$ be the bumped node sequence for the insertion $(\mathrm{T} \stackrel{\varepsilon}{\leftarrow} x)$. If $i<j$ and $\left(b_{i}\right)_{\delta}<\left(b_{j}\right)_{\delta}$, then there exists a sequence $i \leqslant t_{0}<\cdots<t_{k}<j$, where $k=\left(b_{j}\right)_{\delta}-\left(b_{i}\right)_{\delta}-1$, such that $\left(b_{t_{a}}\right)_{\delta}=\left(b_{i}\right)_{\delta}+a$, and $\varepsilon+\overline{\mathrm{T}\left(b_{t_{a}}\right)}=\delta$ for all $a$.

Proof. Let $l$ be minimal such that $\left(b_{i+l}\right)_{\delta} \geqslant\left(b_{i}\right)_{\delta}+1$. Then $i<l \leqslant j$. If $\varepsilon+\overline{\mathrm{T}\left(b_{i+l-1}\right)}=\delta+1$, then the algorithm implies that $\left(b_{i+l}\right)_{\delta} \leqslant\left(b_{i+l-1}\right)_{\delta}$, a contradiction of the minimality of $l$. Thus $\varepsilon+\overline{\mathrm{T}\left(b_{i+l-1}\right)}=\delta$, hence $\left(b_{i+l}\right)_{\delta}=\left(b_{i+l-1}\right)_{\delta}+1$, so by minimality of $l$, we must have $\left(b_{i+l-1}\right)_{\delta}=\left(b_{i}\right)_{\delta}$. Set $t_{0}=i+l-1$. Then $i \leqslant t_{0}<t_{0}+1 \leqslant j$, and $\left(b_{t_{0}+1}\right)_{\delta}=\left(b_{i}\right)_{\delta}+1$. Now the claim follows by induction.

Lemma 16. Let $\mathrm{T}$ be a semistandard $(\mathscr{X}, \lambda)$-tableau, $\varepsilon \in \mathbb{Z}_{2}$, and $x \in \mathscr{X}$. Let $b_{1}, \ldots, b_{m}$ be the bumped node sequence for the insertion $(\mathrm{T} \stackrel{\varepsilon}{\leftarrow} x)$. Let $i, j, k$ be such that

(i) $i, j<k$,

(ii) $b_{i} \not b_{k} \not 7 b_{j}$,

(iii) $\left(\left(b_{i}\right)_{\overline{0}},\left(b_{j}\right)_{\overline{1}}-1\right),\left(\left(b_{i}\right)_{\overline{0}}-1,\left(b_{j}\right)_{\overline{1}}\right) \in[\operatorname{sh}(\mathrm{T} \stackrel{\varepsilon}{\leftarrow} x)]$.

Then there exists some $l>k$ such that

(i) $b_{i} \Rightarrow b_{l}$ and $b_{l} \gg b_{i}$, or;

(ii) $b_{j} \Downarrow b_{l}$ and $b_{i} \pi b_{l}$.

Proof. We will call a triple $(i, k, j)$ which satisfies (i)-(iii) a stair triple. For compactness we'll write $r_{a}$ for $\left(b_{a}\right)_{\overline{0}}$ and $c_{a}$ for $\left(b_{a}\right)_{\overline{1}}$. Define:

$$
\begin{aligned}
m_{i, j, k} & :=\left(c_{j}-c_{k}\right)+\left(r_{i}-r_{k}\right) \\
n_{i, j} & :=\left(c_{j}-c_{i}\right)+\left(r_{i}-r_{j}\right)
\end{aligned}
$$


Note that $2 \leqslant m_{i, j, k} \leqslant n_{i, j}-2$.

Take $n_{i, j}=4$, the least possible value for $n_{i, j}$. Then $m_{i, j, k}=2$. Then $b_{k}=\left(r_{i}-1, c_{j}-1\right)$, so $b_{k} \in[\lambda]$ and thus cannot be the last bumped node. By Lemma 11, either $b_{k+1}=\left(r_{i}, c_{i+1}\right)$ (if $\left.\varepsilon+\overline{\mathrm{T}\left(b_{i}\right)}=\overline{0}\right)$ or $b_{k+1}=\left(r_{j}+1, c_{j}\right)\left(\right.$ if $\left.\varepsilon+\overline{\mathrm{T}\left(b_{i}\right)}=\overline{1}\right)$. Taking $l=k+1$, this completes the base case.

We argue by induction. Assume that $i, j, k$ satisfy (i)-(iii), and further assume that the claim holds for all $i^{\prime}, j^{\prime}, k^{\prime}$ such that $n_{i^{\prime}, j^{\prime}}<n_{i, j}$, or $n_{i^{\prime}, j^{\prime}}=n_{i, j}$ and $m_{i^{\prime}, j^{\prime}, k^{\prime}}<m_{i, j, k}$.

Assume $\varepsilon+\overline{\mathrm{T}\left(b_{k}\right)}=\overline{0}$ (the argument in the other case is exactly dual to what follows). Then $r_{k+1}=r_{k}+1$. If $r_{k+1}=r_{j}$, then, taking $l=k+1$, we are in case (i). Assume $r_{k+1}<r_{i}$. If $c_{k+1}=c_{k}$, we may apply the induction assumption to the stair triple $(i, k+1, j)$. Thus assume $c_{k+1}<c_{k}$. Since $k+1>j$, it must be that $c_{k+1}>c_{j}$. Now, apply the induction assumption to the stair triple $(i, k+1, k)$. This either gives a node $b_{l}$ which satisfies (i), or $b_{l}$ is such that $c_{l}=c_{k}$ and $r_{k}<r_{l}<r_{i}$. In the former case we are done, so assume the latter. Now apply the induction assumption to the stair triple $(i, l, j)$, and we are done.

\subsection{Bumped nodes in successive insertions}

In this section we prove a key result which bounds the distribution of bumped nodes appearing in successive insertions. Theorem 18, together with Corollary 19 can be viewed as a generalization of $[\mathrm{K}$, Theorem 1] to the realm of superalphabets and $\varepsilon$-insertion. We begin by defining a certain set partition of the nodes of a Young diagram that naturally results from the $\varepsilon$-insertion process.

Let $y \in \mathscr{X}, \varepsilon \in \mathbb{Z}_{2}$, and let $\mathrm{T}$ be a semistandard $(\mathscr{X}, \lambda)$-tableau. Let $b_{1}^{y}, \ldots, b_{m}^{y}$ be the bumped nodes for the insertion $(\mathrm{T} \stackrel{\varepsilon}{\leftarrow} y)$. Assume $\operatorname{sh}(\mathrm{T} \stackrel{\varepsilon}{\leftarrow} y)=\mu$. Let $r_{i}^{y}=\left(b_{i}^{y}\right)_{\overline{0}}$ and $c_{i}^{y}=\left(b_{i}^{y}\right)_{\overline{1}}$ for and $1 \leqslant i \leqslant m$. For arbitrary $v \in \mathrm{N}$ we set

$$
\begin{aligned}
l(v) & =\max \left[\left\{j \mid r_{j}^{y}=v_{\overline{0}}, c_{j}^{y}<v_{\overline{1}}\right\} \cup\{0\}\right] \\
u(v) & =\max \left[\left\{j \mid c_{j}^{y}=v_{\overline{1}}, r_{j}^{y}<v_{\overline{0}}\right\} \cup\{0\}\right] .
\end{aligned}
$$

Then $b_{l(v)}^{y}$ is the nearest node directly to the left of $v$ which was bumped in the $y$ insertion. If no such element exists then $l(v)=0$. Similarly, $b_{u(v)}^{y}$ is the nearest node directly above $v$ which was bumped in the $y$ insertion. If no such element exists then $u(v)=0$. Let $[\mu]_{A}$ be the set of all nodes of $[\mu]$ together with all addable nodes of $[\mu]$.

Now define the sets

$$
\begin{aligned}
\mathrm{NE}(\mathrm{T}, \varepsilon, y) & :=\left\{v \in[\mu]_{A} \mid l(v)>u(v)\right\} \cup\left\{v \in[\mu]_{A} \mid l(v)=u(v)=0, \varepsilon+\bar{y}=\overline{1}\right\} \\
\mathrm{SW}(\mathrm{T}, \varepsilon, y) & :=\left\{v \in[\mu]_{A} \mid l(v)<u(v)\right\} \cup\left\{v \in[\mu]_{A} \mid l(v)=u(v)=0, \varepsilon+\bar{y}=\overline{0}\right\}
\end{aligned}
$$

Remark 17. Informally, $\mathrm{NE}(\mathrm{T}, \varepsilon, y)$ represents the set of nodes 'northeast' of a rough perimeter delineated by tracing the path of the bumped nodes in sequence, and $\mathrm{SW}(\mathrm{T}, \varepsilon, y)$ 
represents the nodes to the 'southwest' of that perimeter. For example, if $\varepsilon+\bar{y}=\overline{1}$, and the bumped nodes are those labeled in the diagram of $[\mu]$ below, then the red-colored nodes represent the set $\mathrm{NE}(\mathrm{T}, \varepsilon, y)$, and the blue-colored nodes represent the set $\mathrm{SW}(\mathrm{T}, \varepsilon, y)$.

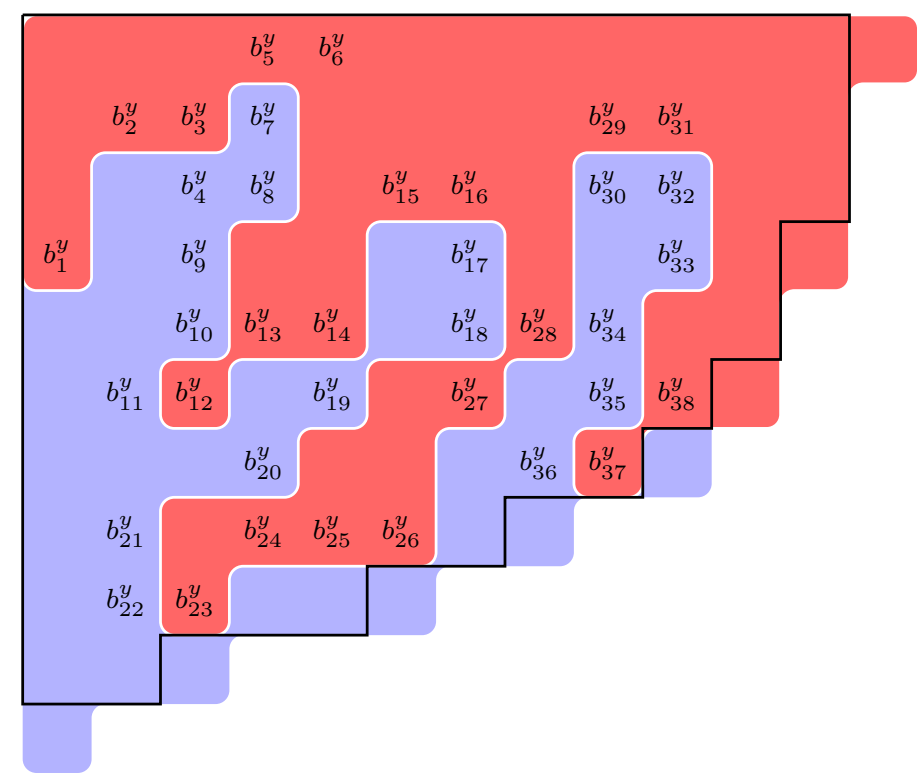

Though we will not need this fact, it follows from the definition that $b_{i}^{y} \in \mathrm{NE}(\mathrm{T}, \varepsilon, y)$ if and only if $\overline{(\mathrm{T} \stackrel{\varepsilon}{\leftarrow} y)\left(b_{i}^{y}\right)}+\varepsilon=\overline{1}$, as can be verified in the example above.

Theorem 18. Assume $\varepsilon \in \mathbb{Z}_{2}, y, z \in \mathscr{X}$, and $\mathrm{T}$ is a semistandard $(\mathscr{X}, \lambda)$-tableau. Let $b_{1}^{y}, \ldots, b_{m_{1}}^{y}$ and $b_{1}^{z}, \ldots, b_{m_{2}}^{z}$ be the bumped node sequences for the insertions $\mathrm{T}_{y}:=(\mathrm{T} \stackrel{\varepsilon}{\leftarrow} y)$ and $\mathrm{T}_{z}:=\left(\mathrm{T}_{y} \stackrel{\varepsilon}{\leftarrow} z\right)$ respectively. Then

$$
\left\{b_{1}^{z}, \ldots, b_{m_{2}}^{z}\right\} \subseteq \mathrm{NE}(\mathrm{T}, \varepsilon, y) \Longleftrightarrow\left\{\begin{array}{l}
y \prec z \text { and } \varepsilon=\overline{0}, \text { or } \\
y \succ z \text { and } \varepsilon=\overline{1}, \text { or } \\
y=z \text { and } \bar{y}=\overline{0},
\end{array}\right.
$$

and

$$
\left\{b_{1}^{z}, \ldots, b_{m_{2}}^{z}\right\} \subseteq \mathrm{SW}(\mathrm{T}, \varepsilon, y) \Longleftrightarrow\left\{\begin{array}{l}
y \succ z \text { and } \varepsilon=\overline{0}, \text { or } \\
y \prec z \text { and } \varepsilon=\overline{1} \text {, or } \\
y=z \text { and } \bar{y}=\overline{1} \text {. }
\end{array}\right.
$$

Proof. Since $\mathrm{NW}(\mathrm{T}, \varepsilon, y) \sqcup \mathrm{SE}(\mathrm{T}, \varepsilon, y)=[\mu]_{A}$, we may prove the equivalent statement:

$$
b_{i}^{z} \in \mathrm{NE}(\mathrm{T}, \varepsilon, y) \Longleftrightarrow\left\{\begin{array}{l}
y \prec z \text { and } \varepsilon=\overline{0}, \text { or } \\
y \succ z \text { and } \varepsilon=\overline{1}, \text { or } \\
y=z \text { and } \bar{y}=\overline{0},
\end{array}\right.
$$


for all $1 \leqslant i \leqslant m_{2}$. First we prove that the lemma holds when $\mathrm{T}$ is a standard tableau, $y, z \notin \mathrm{T}, y \neq z$, and $\bar{y}=\overline{0}$. Note that in this situation we need not consider the third case in the right side of (4.1). We will go by induction on $1 \leqslant i \leqslant m_{2}$. We will write NE for $\mathrm{NE}(\mathrm{T}, \varepsilon, y)$ where the context is clear.

Base case $i=1$. Assume $y \prec z$. Since $\bar{y}=\overline{0}$, we have $y<z$ and $\bar{z}=\overline{0}$. If $\varepsilon=\overline{0}$, then $b_{1}^{y}$ is in the first row, and $\mathrm{T}_{y}\left(b_{1}^{y}\right)=y<z$, so $b_{1}^{y} \Rightarrow b_{1}^{z}$. Then $l_{1}>0=u_{1}$, so $b_{1}^{z} \in$ NE. If $\varepsilon=\overline{1}$, then $b_{1}^{y}$ is in the first column, and $b_{1}^{z} \Uparrow b_{1}^{y}$ so $l_{1}=0<u_{1}$, so $b_{i}^{z} \notin \mathrm{NE}$.

Now assume $y \succ z$ and $\varepsilon=\overline{0}$. Then $b_{1}^{y}$ is in the first row. If $\bar{z}=\overline{0}$, then $z<y$, so $b_{1}^{z} \Rightarrow b_{1}^{y}$. Then $l_{1}=0$, so $b_{1}^{z} \notin \mathrm{NE}$. If $\bar{z}=\overline{1}$, then $b_{1}^{z}$ is in the first column, so $l_{1}=0$, and again $b_{1}^{z} \notin \mathrm{NE}$.

Now assume $y \succ z$ and $\varepsilon=\overline{1}$. Then $b_{1}^{y}$ is in the first column. If $\bar{z}=\overline{0}$, then $z<y$, so $b_{1}^{y} \Uparrow b_{1}^{z}$. Then $u_{1}=0$, so $b_{1}^{z} \in \mathrm{NE}$. If $\bar{z}=\overline{1}$, then $b_{1}^{z}$ is in the first row, so again $u_{1}=0$ and $b_{1}^{z} \in \mathrm{NE}$.

Induction step. So (4.1) holds when $i=1$. Now we show that

$$
b_{i}^{z} \in \mathrm{NE} \quad \Longleftrightarrow \quad b_{i+1}^{z} \in \mathrm{NE} .
$$

( $\Longrightarrow$ ) Assume $b_{i}^{z} \in \mathrm{NE}$. Then $l_{i}>u_{i}$, or $l_{i}=u_{i}=0$ and $\varepsilon=\overline{1}$. We assume the former, and will later address the latter case. If $b_{k}^{y} \neq b_{u_{i}}^{y}$ is a node such that $b_{k}^{y} \searrow b_{u_{i}}^{y}$, then $k<u_{i}$. By Lemma 16, if $b_{k}^{y}$ is a node such that $b_{l_{i}}^{y} \not b_{k}^{y} \not b_{u_{i}}^{y}$, then $k<l_{i}$. From this it follows that $\varepsilon+\mathrm{T}\left(b_{l_{i}}^{y}\right)=\overline{0}$, so $\left(b_{l_{i}+1}^{y}\right)_{\overline{0}}=\left(b_{l_{i}}^{y}\right)_{\overline{0}}+1$. There are two cases to consider:

(a) Assume $\varepsilon+\overline{\mathrm{T}_{y}\left(b_{i}^{z}\right)}=\overline{0}$. Then $\left(b_{i+1}^{z}\right)_{\overline{0}}=\left(b_{i}^{z}\right)_{\overline{0}}+1$. First we show that $b_{i}^{z} \neq b_{r}^{y}$ for any $r$. Indeed, if we did have that $b_{i}^{z}=b_{r}^{y}$, then by Lemma 15 applied to $\left(b_{l_{i}}^{y}, b_{r}^{y}\right)$, there is some $b_{s}^{y}$ in the column to the left of $b_{r}^{y}$, with $l_{i} \leqslant s<i$ and $\varepsilon+\overline{\mathrm{T}\left(b_{s}^{y}\right)}=\overline{1}$. Then by the above paragraph, $b_{s}^{y} \nearrow b_{i}^{y}$. But then $b_{s+1}^{y}$ is in the same column as $b_{i}^{y}$, but cannot be above or below $b_{i}^{y}$ since $u_{i}<s<s+1 \leqslant i$. Then the only option is $s+1=r$. But this cannot be, since by assumption

$$
\varepsilon+\overline{\mathrm{T}\left(b_{s}^{y}\right)}=\varepsilon+\overline{\mathrm{T}\left(b_{r-1}^{y}\right)}=\varepsilon+\overline{\mathrm{T}_{y}\left(b_{r}^{y}\right)}=\varepsilon+\overline{\mathrm{T}_{y}\left(b_{i}^{z}\right)}=\overline{0},
$$

a contradiction. So $b_{i}^{z} \neq b_{r}^{y}$, thus $\mathrm{T}_{y}\left(b_{i}^{z}\right)=\mathrm{T}\left(b_{i}^{z}\right)$ and $u_{i+1} \leqslant l_{i}$.

We have $\left(b_{l_{i}+1}^{y}\right)_{\overline{0}}=\left(b_{l_{i}}^{y}\right)_{\overline{0}}+1$, so $\left(b_{l_{i}+1}^{y}\right)_{\overline{0}}=\left(b_{i+1}^{z}\right)_{\overline{0}}$. Moreover, since $\mathrm{T}_{y}\left(b_{l_{i}+1}^{y}\right)=$ $\mathrm{T}\left(b_{l_{i}}^{y}\right)<\mathrm{T}\left(b_{i}^{z}\right)=\mathrm{T}_{y}\left(b_{i}^{z}\right)=x_{i+1}^{z}$, we have that $b_{l_{i}+1}^{y} \Rightarrow b_{i+1}^{z}$, so $l_{i+1} \geqslant l_{i}+1>l_{i} \geqslant u_{i+1}$. Thus $b_{i+1}^{z} \in \mathrm{NE}$.

(b) Assume $\varepsilon+\overline{\mathrm{T}_{y}\left(b_{i}^{z}\right)}=\overline{1}$. Note $\mathrm{T}_{y}\left(b_{i}^{z}\right)=\mathrm{T}_{z}\left(b_{i+1}^{z}\right)$. We have $\left(b_{i+1}^{z}\right)_{\overline{1}}=\left(b_{i}^{z}\right)_{\overline{1}}+1$. First we prove that $u_{i+1}<l_{i}$. Assume this is not the case. Then by Lemma 15, there exists a sequence $t_{0}, \ldots, t_{k}$, where $k=\left(b_{u_{i+1}}^{y}\right)_{\overline{1}}-\left(b_{l_{i}}^{y}\right)_{\overline{1}}-1$, such that $l_{i} \leqslant t_{0}<\cdots<t_{k}<u_{i+1}$, $\left(b_{t_{j}}^{y}\right)_{\overline{1}}=\left(b_{l_{i}}^{y}\right)_{\overline{1}}+j$ and $\varepsilon+\overline{\mathrm{T}\left(b_{t_{j}}^{y}\right)}=\overline{1}$ for all $j$. Then $b_{t_{k}}^{y}$ is in the same column as $b_{i}^{z}$, and $u_{i}<l_{i} \leqslant t_{k}$, so we must have $b_{i}^{z} \downarrow b_{t_{k}}^{y}$. Then $x_{i+1}^{z}=\mathrm{T}_{y}\left(b_{i}^{z}\right) \leqslant \mathrm{T}_{y}\left(b_{t_{k}}^{y}\right)<\mathrm{T}_{y}\left(b_{u_{i+1}}^{y}\right)$, a contradiction of the $\varepsilon$-insertion algorithm. Thus $u_{i+1}<l_{i}$. 
If $u_{i+1}=0$, then $b_{i+1}^{z} \in \mathrm{NE}$ unless $l_{i+1}=0$ and $\varepsilon=\overline{0}$. We rule out this case by way of contradiction. Let $u_{i+1}=l_{i+1}=0$ and $\varepsilon=\overline{0}$. Then by Lemma 16, there is no $m$ such that $b_{m}^{y} \searrow b_{i+1}^{z}$. Then $b_{i+1}^{z} \nearrow b_{1}^{y}$. Thus there is a sequence $t_{0}, \ldots, t_{k}$, where $k=\left(b_{l_{i}}^{y}\right)_{\overline{0}}-2$ such that $1 \leqslant t_{0}<\cdots<t_{k}<l_{i},\left(b_{t_{j}}^{y}\right)_{\overline{0}}=1+j$ and $\varepsilon+\overline{\mathrm{T}\left(b_{t_{j}}^{y}\right)}=\overline{0}$ for all $j$. Writing $t_{k+1}:=l_{i}$, there is some $t_{a}$ such that $b_{t_{a}}^{y}$ is in the same row as $b_{i+1}^{z}$. But then either $b_{i+1}^{z}=b_{t_{a}}^{y}$ (in which case $x_{i+1}^{z}<\mathrm{T}_{y}\left(b_{t_{a}}^{y}\right)$ ), or $b_{i+1}^{z} \Rightarrow b_{t_{a}}^{y}$ (in which case $\left.x_{i+1}^{z}<\mathrm{T}_{y}\left(b_{i+1}^{z}\right)<\mathrm{T}_{y}\left(b_{t_{a}}^{y}\right)\right)$. But we also have $x_{i+1}^{z}=\mathrm{T}_{y}\left(b_{i}^{z}\right)>\mathrm{T}_{y}\left(b_{l_{i}}^{y}\right) \geqslant \mathrm{T}_{y}\left(b_{t_{a}}^{y}\right)$, a contradiction of the $\varepsilon$-insertion algorithm.

So assume $u_{i+1}>0$. Then by Lemma 15 there exists a sequence $t_{0}, \ldots, t_{k}$, where $k=\left(b_{l_{i}}^{y}\right)_{\overline{0}}-\left(b_{u_{i+1}}\right)_{\overline{0}}-1$, such that $u_{i+1} \leqslant t_{0}<\cdots<t_{k}<l_{i},\left(b_{t_{j}}^{y}\right)_{\overline{0}}=\left(b_{u_{i+1}}\right)_{\overline{0}}+j$ and $\varepsilon+\overline{\mathrm{T}\left(b_{t_{j}}^{y}\right)}=\overline{0}$ for all $j$. If $b_{i}^{z} \Rightarrow b_{i+1}^{z}$, then $l_{i+1} \geqslant l_{i}$. Otherwise there is some $0 \leqslant a \leqslant k$ such that $b_{t_{a}}^{y}$ is in the same row as $b_{i+1}^{z}$. But since $\mathrm{T}_{y}\left(b_{i+1}^{z}\right)>\mathrm{T}_{y}\left(b_{i}^{z}\right)>$ $\mathrm{T}_{y}\left(b_{l_{i}}^{y}\right)>\mathrm{T}_{y}\left(b_{t_{a}}^{y}\right)$, it must be that $b_{t_{a}}^{y} \Rightarrow b_{i+1}^{z}$. Then $l_{i+1} \geqslant t_{a} \geqslant u_{i+1}$, and thus $l_{i+1}>u_{i+1}$, so $b_{i+1}^{z} \in \mathrm{NE}$.

Now assume that $u_{i}=l_{i}=0$ and $\varepsilon=\overline{1}$. We will show that $u_{i+1}=0$. By way of contradiction assume $u_{i+1}>0$. There are two cases to consider:

(a) Assume $\overline{\mathrm{T}_{y}\left(b_{i}^{z}\right)}=\overline{0}$. Then, since $b_{1}^{y}$ is in the first column, by Lemma 15 there exists a sequence $t_{0}, \ldots, t_{k}$, where $k=\left(b_{u_{i+1}}^{y}\right)_{\overline{1}}-2$, such that $1 \leqslant t_{0}<\cdots<t_{k}<u_{i+1}$, $\left(b_{t_{j}}^{y}\right)_{\overline{1}}=1+j$ and $\varepsilon+\overline{\mathrm{T}\left(b_{t_{j}}^{y}\right)}=\overline{1}$ for all $j$. Then $b_{i}^{z} \downarrow b_{t_{k}}^{y}$. Then $x_{i+1}^{z}=\mathrm{T}_{y}\left(b_{i}^{z}\right) \leqslant$ $\mathrm{T}_{y}\left(b_{t_{k}}^{y}\right)<\mathrm{T}_{y}\left(b_{u_{i+1}}^{y}\right)$, so by $\overline{1}$-insertion, $b_{i+1}^{z} \downarrow b_{u_{i+1}}^{y}$, a contradiction.

(b) Assume $\overline{\mathrm{T}_{y}\left(b_{i}^{z}\right)}=\overline{1}$. Then, applying Lemma 15, we have $u_{i+1}=0$ unless $b_{i}^{z} \Downarrow b_{i+1}^{z}$ and $b_{i}^{z}=b_{r}^{y}$ for some $r$. Then $\mathrm{T}\left(b_{r-1}^{y}\right)=\mathrm{T}_{y}\left(b_{r}^{y}\right)$, and $\overline{\mathrm{T}_{y}\left(b_{r}^{y}\right)}=\overline{1}$, so $b_{r-1}^{y}$ is in the row above $b_{r}^{y}$, and $b_{r}^{y} \rrbracket b_{r-1}^{y}$. Then, since $b_{1}^{y}$ is in the first column, by Lemma 15 there exists a sequence $t_{0}, \ldots, t_{k}$, where $k=\left(b_{r-1}^{y}\right)_{\overline{1}}-2$, such that $1 \leqslant t_{0}<\cdots<t_{k}<r-1$, $\left(b_{t_{j}}^{y}\right)_{\overline{1}}=1+j$ and $\overline{\mathrm{T}\left(b_{t_{j}}^{y}\right)}=\overline{0}$ for all $j$. Then there exist some $t_{j}$ such that $b_{t_{j}}^{y}$ is in the same column as $b_{r}^{y}$. Then, since $t_{j}<r$, we have $b_{r}^{y} \Uparrow b_{t_{j}}^{y}$, a contradiction, since $u_{i}=0$.

This completes the proof that $b_{i+1}^{z} \in \mathrm{NE}$ if $b_{i}^{z} \in \mathrm{NE}$.

$(\Longleftarrow)$ Now assume $b_{i}^{z} \notin \mathrm{NE}(\mathrm{T}, \varepsilon, y)$. Let $c_{1}^{y}, \ldots, c_{m_{1}}^{y}$ and $c_{1}^{z}, \ldots, c_{m_{2}}^{z}$ be the bumped node sequences for the insertions $\left(\mathrm{T}^{\prime} \stackrel{\varepsilon+\overline{1}}{\longleftarrow} y\right)$ and $\left(\left(\mathrm{T}^{\prime} \stackrel{\varepsilon+\overline{1}}{\longleftarrow} y\right) \stackrel{\varepsilon+\overline{1}}{\longleftarrow} z\right)$, respectively. Then by Lemma $2, c_{j}^{y}=\left(b_{j}^{y}\right)^{\prime}$ and $c_{j}^{z}=\left(b_{j}^{z}\right)^{\prime}$ for all $j$. But then $u\left(b_{j}^{z}\right)=l\left(c_{j}^{z}\right)$ and $l\left(b_{j}^{z}\right)=u\left(c_{j}^{z}\right)$ for all $j$, so $c_{i}^{z} \in \mathrm{NE}\left(\mathrm{T}^{\prime}, \varepsilon+\overline{1}, y\right)$. Then, applying the 'only if' direction of the claim proved above, we have $c_{i+1}^{z} \in \mathrm{NE}\left(\mathrm{T}^{\prime}, \varepsilon+\overline{1}, y\right)$. Then

$$
u\left(b_{j}^{z}\right)=l\left(c_{j}^{z}\right)>u\left(c_{j}^{z}\right)=l\left(b_{j}^{z}\right), \quad \text { or } \quad u\left(b_{j}^{z}\right)=l\left(c_{j}^{z}\right)=0=u\left(c_{j}^{z}\right)=l\left(b_{j}^{z}\right) \text { and } \varepsilon+\bar{y}=\overline{0},
$$

so $b_{i+1}^{z} \notin \mathrm{NE}(\mathrm{T}, \varepsilon, y)$, as required. 
This completes the proof of the lemma when $\mathrm{T}$ is a standard tableau, $y, z \notin \mathrm{T}, y \neq z$, and $\bar{y}=\overline{0}$. Now we maintain the above assumptions but consider the case $\bar{y}=\overline{1}$. Let $c_{1}^{y^{*}}, \ldots, c_{m_{1}}^{y^{*}}$ and $c_{1}^{z^{*}}, \ldots, c_{m_{2}}^{z^{*}}$ be the bumped node sequences for the insertions $\left(\mathrm{T}^{*} \stackrel{\varepsilon+\overline{1}}{\longleftarrow} y^{*}\right)$ and $\left(\left(\mathrm{T}^{*} \stackrel{\varepsilon+\overline{1}}{\longleftarrow} y^{*}\right) \stackrel{\varepsilon+\overline{1}}{\longleftarrow} z^{*}\right)$, respectively. Then $b_{i}^{y}=c_{i}^{y^{*}}$ and $b_{i}^{z}=c_{i}^{z^{*}}$ for all $i$, so $u\left(b_{i}^{z}\right)=$ $u\left(c_{i}^{z^{*}}\right)$ and $l\left(b_{i}^{z}\right)=l\left(c_{i}^{z^{*}}\right)$ for all $i$ by Lemma 2 . Then, since $\overline{y^{*}}=\overline{0}$, we have that, for all $i$,

$$
\begin{aligned}
b_{i}^{z} \in \mathrm{NE}(\mathrm{T}, \varepsilon, y) & \Longleftrightarrow \quad c_{i}^{z^{*}} \in \mathrm{NE}\left(\mathrm{T}^{*}, \varepsilon+\overline{1}, y^{*}\right) \\
& \Longleftrightarrow\left\{\begin{array}{l}
y^{*} \prec z^{*} \text { and } \varepsilon+\overline{1}=\overline{0}, \text { or } \\
y^{*} \succ z^{*} \text { and } \varepsilon+\overline{1}=\overline{1}
\end{array}\right. \\
& \Longleftrightarrow\left\{\begin{array}{l}
y \succ z \text { and } \varepsilon=\overline{1}, \text { or } \\
y \prec z \text { and } \varepsilon=\overline{0} .
\end{array}\right.
\end{aligned}
$$

This completes the proof of the lemma when $\mathrm{T}$ is a standard tableau, $y, z \notin \mathrm{T}, y \neq z$.

Now, let $\mathrm{T}$ be an arbitrary semistandard tableau, with arbitrary $y, z \in \mathscr{X}$. We may choose elements $z^{\bullet}, y^{\bullet} \in \mathscr{X}^{\bullet}$, and a $\bullet$-standardization $\mathrm{T}^{\bullet}$ of $\mathrm{T}$, such that

(i) $\hat{\bullet}\left(z^{\bullet}\right)=z$

(ii) $\hat{\bullet}\left(y^{\bullet}\right)=y$

(iii) For all $x \in \mathrm{T}^{\bullet}$ such that $y=\hat{\bullet}(x)$, we have:

(a) $x \prec y^{\bullet}$ if $\varepsilon+\bar{y}=\overline{0}$

(b) $x \succ y^{\bullet}$ if $\varepsilon+\bar{y}=\overline{1}$

(iv) For all $x \in \mathrm{T}^{\bullet}$ such that $z=\hat{\bullet}(x)$, we have:

(a) $x \prec z^{\bullet}$ if $\varepsilon+\bar{z}=\overline{0}$

(b) $x \succ z^{\bullet}$ if $\varepsilon+\bar{z}=\overline{1}$

(v) If $z=y$, we have:

(a) $y^{\bullet} \prec z^{\bullet}$ if $\varepsilon+\bar{z}=\overline{0}$

(b) $y^{\bullet} \succ z^{\bullet}$ if $\varepsilon+\bar{z}=\overline{1}$.

Then by this choice we have

$$
\begin{aligned}
b_{i}^{z} \in \mathrm{NE}(\mathrm{T}, \varepsilon, y) & \Longleftrightarrow \quad b_{i}^{z^{\bullet}} \in \mathrm{NE}\left(\mathrm{T}^{\bullet}, \varepsilon, y^{\bullet}\right) \\
& \Longleftrightarrow\left\{\begin{array}{l}
y^{\bullet} \prec z^{\bullet} \text { and } \varepsilon=\overline{0}, \text { or } \\
y^{\bullet} \succ z^{\bullet} \text { and } \varepsilon=\overline{1}
\end{array}\right. \\
& \Longleftrightarrow\left\{\begin{array}{l}
y \prec z \text { and } \varepsilon=\overline{0}, \text { or } \\
y \succ z \text { and } \varepsilon=\overline{1}, \text { or } \\
y=z \text { and } \bar{y}=\overline{0},
\end{array}\right.
\end{aligned}
$$


by application of Lemma 14 .

Corollary 19. Assume $\varepsilon \in \mathbb{Z}_{2}, y, z \in \mathscr{X}$, and $\mathrm{T}$ is a semistandard $(\mathscr{X}, \lambda)$-tableau. Then

$$
A(\mathrm{~T}, \varepsilon, y) \nearrow A((\mathrm{~T} \stackrel{\varepsilon}{\leftarrow} y), \varepsilon, z) \Longleftrightarrow\left\{\begin{array}{l}
y \prec z \text { and } \varepsilon=\overline{0}, \text { or } \\
y \succ z \text { and } \varepsilon=\overline{1}, \text { or } \\
y=z \text { and } \bar{y}=\overline{0} .
\end{array}\right.
$$

Proof. Let $b_{1}^{y}, \ldots, b_{m_{1}}^{y}, b_{1}^{z}, \ldots, b_{m_{2}}^{z}$ be as in Lemma 18. By that lemma, $b_{m_{2}}^{z} \in \mathrm{NE}(\mathrm{T}, \varepsilon, y)$ if and only if the right side holds.

$\left(\Longleftarrow\right.$ ) Assume by way of contradiction that $b_{m_{2}}^{z} \in \mathrm{NE}$ and $b_{m_{2}}^{z} \nearrow b_{m_{1}}^{y}$. Then $b_{m_{1}}^{y}$ cannot be in the same column as $b_{m_{2}}^{z}$, else $u_{m_{2}}>l_{m_{2}}$.

First assume $l_{m_{2}}>0$. Then by Lemma 15, there exists a sequence $t_{0}, \ldots, t_{k}$, where $k=\left(b_{m_{1}}^{y}\right)_{\overline{1}}-\left(b_{l_{m_{2}}}^{y}\right)_{\overline{1}}-1$, such that $l_{m_{2}} \leqslant t_{0}<\cdots<t_{k}<m_{1},\left(b_{t_{j}}^{y}\right)_{\overline{1}}=\left(b_{l_{m_{2}}}^{y}\right)_{\overline{1}}+j$ and $\varepsilon+\overline{\mathrm{T}\left(b_{t_{j}}^{y}\right)}=\overline{1}$ for all $j$. Then there is some $t_{j}$ such that $b_{t_{j}}^{y}$ is in the same column as $b_{m_{2}}^{z}$. Moreover, we have $b_{m_{2}}^{z} \Uparrow b_{t_{j}}^{y}$, hence $u_{m_{2}} \geqslant t_{j}>l_{m_{2}}$, a contradiction.

Now assume $l_{m_{2}}=0$. Then $u_{m_{2}}=0$ and $\varepsilon+\bar{y}=\overline{1}$. Then $b_{1}^{y}$ is in the first column, and by Lemma 15 , there exists a sequence $t_{0}, \ldots, t_{k}$, where $k=\left(b_{m_{1}}^{y}\right)_{\overline{1}}-2$, such that $1 \leqslant t_{0}<\cdots<t_{k}<m_{1},\left(b_{t_{j}}^{y}\right)_{\overline{1}}=1+j$ and $\varepsilon+\overline{\mathrm{T}\left(b_{t_{j}}^{y}\right)}=\overline{1}$ for all $j$. Then there is some $t_{j}$ such that $b_{t_{j}}^{y}$ is in the same column as $b_{m_{2}}^{z}$. Moreover, we have $b_{m_{2}}^{z} \Uparrow b_{t_{j}}^{y}$, hence $u_{m_{2}} \geqslant t_{j}>0$, a contradiction.

( $\Longrightarrow$ ) Applying the 'if' statement proved above to the conjugate situation (as in the proof of claim Lemma 18), we have that $b_{m_{2}} \notin \mathrm{NE}$ implies that $b_{m_{2}}^{z} \nearrow b_{m_{1}}^{y}$, completing the proof.

\section{Super RSK correspondence}

\subsection{Biwords}

Given alphabets $\mathscr{X}$ and $\mathscr{Y}$, we call an element of $\mathscr{X} \times \mathscr{Y}$ an $(\mathscr{X}, \mathscr{Y})$-biletter. We call a biletter $(x, y)$ mixed if $\bar{x}+\bar{y}=\overline{1}$. We define a total order $\triangleleft$ on $(\mathscr{X}, \mathscr{Y})$-biletters by setting $\left(x_{1}, y_{1}\right) \triangleleft\left(x_{2}, y_{2}\right)$ if

$$
y_{1}<\mathscr{Y} y_{2}, \quad \text { or } \quad y_{1}=y_{2}, x_{1} \prec \mathscr{X} x_{2} \text {. }
$$

For $k \in \mathbb{Z}_{>0}$, we call an element $\boldsymbol{w}=\left(\left(x_{1}, y_{1}\right), \ldots,\left(x_{k}, y_{k}\right)\right) \in(\mathscr{X} \times \mathscr{Y})^{k}$ an $(\mathscr{X}, \mathscr{Y})$ biword of length $k$. We say that $\boldsymbol{w}$ is restricted if it is multiplicity free with respect to mixed biletters; i.e. $\left(x_{i}, y_{i}\right)=\left(x_{j}, y_{j}\right)$ for $i \neq j$ only if $\bar{x}_{i}+\bar{y}_{i}=\overline{0}$. We say that $\boldsymbol{w}$ is ordered if $\left(x_{i}, y_{i}\right) \unlhd\left(x_{j}, y_{j}\right)$ for all $i \leqslant j$. The left content lcon $(\boldsymbol{w})$ of $\boldsymbol{w}$ is the multiset $\left\{x_{1}, \ldots, x_{k}\right\}$ and the right content $\operatorname{rcon}(\boldsymbol{w})$ of $\boldsymbol{w}$ is the multiset $\left\{y_{1}, \ldots, y_{k}\right\}$. 
If $L$ is a multiset of elements of $\mathscr{X}$ and $R$ is a multiset of elements of $\mathscr{Y}$, with $|L|=$ $|R|=k$, we say $(L, R)$ is an $(\mathscr{X}, \mathscr{Y})$-content pair of length $k$. For an $(\mathscr{X}, \mathscr{Y})$-content pair, define $\operatorname{RBiw}(L, R)$ to be the set of restricted $(\mathscr{X}, \mathscr{Y})$-biwords $\boldsymbol{w}$ with $\operatorname{lcon}(\boldsymbol{w})=L$ and $\operatorname{rcon}(\boldsymbol{w})=R$. Let $\operatorname{RBiw}(L, R)^{\unlhd}=\{\boldsymbol{w} \in \operatorname{RBiw}(L, R) \mid \boldsymbol{w}$ is ordered $\}$. Finally, define $\operatorname{Tab}(L, R)$ to be the set of pairs $(\mathrm{L}, \mathrm{R})$ of tableaux such that $\operatorname{sh}(\mathrm{L})=\operatorname{sh}(\mathrm{R}), \operatorname{con}(\mathrm{L})=L$ and $\operatorname{con}(\mathrm{R})=R$. Let $\operatorname{SStd}(L, R) \subseteq \operatorname{Tab}(L, R)$ be the subset of semistandard tableau pairs.

\subsection{Super RSK algorithm}

Let $(L, R)$ be an $(\mathscr{X}, \mathscr{Y})$-content pair of length $k$. Let $\boldsymbol{w}=\left(\left(x_{1}, y_{1}\right), \ldots,\left(x_{k}, y_{k}\right)\right) \in$ $\operatorname{RBiw}(L, R)^{\unlhd}$. We define $\mathrm{T}_{\boldsymbol{w}}^{0}:=\varnothing$, then for $1 \leqslant i \leqslant k$ we inductively define $\mathrm{T}_{\boldsymbol{w}}^{i}:=$ $\left(\mathrm{T}_{\boldsymbol{w}}^{i-1} \stackrel{\bar{y}_{i}}{\longleftarrow} x_{i}\right)$, and define $a_{\boldsymbol{w}}^{i}$ to be the added node of this insertion. We say $\mathrm{T}_{\boldsymbol{w}}:=\mathrm{T}_{\boldsymbol{w}}^{k}$ is the insertion tableau of $\boldsymbol{w}$. The recording tableau of $\boldsymbol{w}$ is the $\left(\mathscr{Y}, \operatorname{sh}\left(\mathrm{T}_{\boldsymbol{w}}\right)\right)$-tableau $\mathrm{T}^{\boldsymbol{w}}$ defined by $\mathrm{T}^{\boldsymbol{w}}\left(a_{\boldsymbol{w}}^{i}\right):=y_{i}$. We then define

$$
\operatorname{sRSK}(\boldsymbol{w}):=\left(\mathrm{T}_{\boldsymbol{w}}, \mathrm{T}^{\boldsymbol{w}}\right) .
$$

Example 20. Let $\mathscr{X}$ be as in Example 3, and take $\mathscr{Y}=\mathscr{X}$. Let

$$
L=\{\hat{1}, 1, \hat{2}, 2, \hat{3}, \hat{3}, \hat{3}, 3\} \quad \text { and } \quad R=\{\hat{1}, \hat{2}, 2,2, \hat{3}, \hat{3}, 3,3\}
$$

be multisets of letters. Then $(L, R)$ is an $(\mathscr{X}, \mathscr{Y})$-content pair of length 8 . Let $\boldsymbol{w}$ be the biword

$$
\boldsymbol{w}=((\hat{3}, \hat{1}),(1, \hat{2}),(2,2),(3,2),(\hat{3}, \hat{3}),(\hat{3}, \hat{3}),(\hat{2}, 3),(\hat{1}, 3)) .
$$

Then $\boldsymbol{w} \in \operatorname{RBiw}(L, R)^{\unlhd}$, and $\operatorname{sRSK}(\boldsymbol{w})$ yields the tableaux:



map sRSK : $\boldsymbol{w} \mapsto\left(\mathrm{T}_{\boldsymbol{w}}, \mathrm{T}^{\boldsymbol{w}}\right)$ defines a bijection $\operatorname{RBiw}(L, R)^{\unlhd} \rightarrow \operatorname{SStd}(L, R)$.

Proof. Let $|L|=|R|=k$, and $\boldsymbol{w}=\left(\left(x_{1}, y_{1}\right), \ldots,\left(x_{k}, y_{k}\right)\right) \in \operatorname{RBiw}(L, R)^{\unlhd}$. We have that $\mathrm{T}_{\boldsymbol{w}}$ is semistandard by inductive application of Lemma 12 . Define $\mathrm{T}_{i}^{\boldsymbol{w}}$ by $\mathrm{T}_{i}^{\boldsymbol{w}}\left(a_{\boldsymbol{w}}^{j}\right):=y_{j}$ for all $1 \leqslant j \leqslant i$. By induction, assume:

(i) $\mathrm{T}_{i}^{w}$ is semistandard

(ii) If $r<s \leqslant i, y_{r}=y_{s}$, and $\bar{y}_{r}=\overline{0}$, then $a_{\boldsymbol{w}}^{r} \nearrow a_{\boldsymbol{w}}^{s}$

(iii) If $r<s \leqslant i, y_{r}=y_{s}$, and $\bar{y}_{r}=\overline{1}$, then $a_{\boldsymbol{w}}^{s} \nearrow a_{\boldsymbol{w}}^{r}$. 
If $y_{i+1}>y_{i}$, then $\mathrm{T}_{i+1}^{\boldsymbol{w}}$ automatically satisfies (i)-(iii). Assume $y_{i+1}=y_{i}$. Then either $x_{i} \prec x_{i+1}$ or $x_{i}=x_{i+1}$ and $\bar{y}_{i+1}+\bar{x}_{i+1}=\overline{0}$. Note that $\mathrm{T}_{i+1}^{w}$ is non-decreasing since the upper row of $\boldsymbol{w}$ is non-decreasing. There are two cases:

(a) Assume $\bar{y}_{i+1}=\overline{0}$. Then (iii) holds, and $\mathrm{T}_{i}^{\boldsymbol{w}}$ is column-strict with respect to odd letters. Moreover by Corollary 19, $a_{\boldsymbol{w}}^{i} \nearrow a_{\boldsymbol{w}}^{i+1}$, so (ii) holds, and $\mathrm{T}_{i}^{\boldsymbol{w}}$ is row-strict with respect to even letters.

(b) Assume $\bar{y}_{i+1}=\overline{1}$. Then (ii) holds, and $\mathrm{T}_{i}^{\boldsymbol{w}}$ is row-strict with respect to even letters. Moreover by Corollary $19, a_{\boldsymbol{w}}^{i+1} \nearrow a_{\boldsymbol{w}}^{i}$, so (iii) holds, and $\mathrm{T}_{i}^{\boldsymbol{w}}$ is column-strict with respect to odd letters.

Thus, by induction $\mathrm{T}_{k}^{\boldsymbol{w}}=\mathrm{T}^{\boldsymbol{w}}$ is semistandard. Thus $\operatorname{sRSK}(\boldsymbol{w}) \in \operatorname{SStd}(L, R)$.

Now let $(\mathrm{L}, \mathrm{R}) \in \operatorname{SStd}(L, R)$. We define $\mathrm{L}_{k}=\mathrm{L}, \mathrm{R}_{k}=\mathrm{R}$, and then for $1 \leqslant i \leqslant k$ inductively define $\mathrm{R}_{i-1}$ and $\mathrm{L}_{i-1}$ in the following manner. Define $y_{i} \in \mathscr{Y}$ to be the $<-$ maximal element of $\mathrm{R}_{i}$. If $\bar{y}_{i}=\overline{0}$ (resp. $\left.\bar{y}_{i}=\overline{1}\right)$, let $u_{i}$ be the rightmost (resp. bottommost) node in $\mathrm{R}_{i}$ such that $\mathrm{R}_{i}\left(u_{i}\right)=y_{i}$. Let $\mathrm{R}_{i-1}=\mathrm{R}_{i} \backslash\left\{u_{i}\right\}$. Let $\mathrm{L}_{i-1}=\left(\mathrm{L}_{i} \stackrel{\bar{y}_{i}}{\rightarrow} u_{i}\right)$, and let $x_{i}$ be the extracted letter. Then define

$$
\operatorname{sRSK}^{*}(\mathrm{~L}, \mathrm{R})=\left(\left(x_{1}, y_{1}\right), \ldots,\left(x_{k}, y_{k}\right)\right)
$$

Let $\boldsymbol{w}:=\operatorname{sRSK}^{*}(\mathrm{~L}, \mathrm{R})$. By construction and Lemma 11 we have that $\mathrm{T}_{\boldsymbol{w}}^{j}=\mathrm{L}_{j}, \mathrm{~T}_{j}^{\boldsymbol{w}}=\mathrm{R}_{j}$, and $u_{j}=a_{\boldsymbol{w}}^{j}$ for all $1 \leqslant j \leqslant k$. We argue by induction on $i$ that

$$
\boldsymbol{w}_{i}:=\left(\left(x_{1}, y_{1}\right), \ldots,\left(x_{i}, y_{i}\right)\right)
$$

is an ordered restricted ( $\mathscr{X}, \mathscr{Y})$-biword. By construction, $y_{i+1} \geqslant y_{i}$, so $\boldsymbol{w}_{i+1}$ is an ordered restricted biword if $y_{i+1} \neq y_{i}$. Assume $y_{i}=y_{i+1}$. Then there are two cases.

(a) Assume $\bar{y}_{i+1}=\overline{0}$. If $x_{i} \succ x_{i+1}$ or $x_{i}=x_{i+1}$ and $\bar{x}_{i}=\overline{1}$, then by Corollary 19, $u_{i+1}=$ $a_{\boldsymbol{w}}^{i+1} \nearrow a_{\boldsymbol{w}}^{i}=u_{i}$, which by the choice of $u_{i+1}$ implies that $u_{i} \Downarrow u_{i+1}, \mathrm{R}\left(u_{i}\right)=\mathrm{R}\left(u_{i+1}\right)$, and $\overline{\mathrm{R}\left(u_{i}\right)}=\overline{0}$, a contradiction, since $\mathrm{R}$ is semistandard.

(b) Assume $\bar{x}_{i+1}=\overline{1}$. If $x_{i} \succ x_{i+1}$ or $x_{i}=x_{i+1}$ and $\bar{x}_{i}=\overline{0}$, then by Corollary 19, $u_{i}=a_{\boldsymbol{w}}^{i} \nearrow a_{\boldsymbol{w}}^{i+1}=u_{i+1}$, which by the choice of $u_{i+1}$ implies that $u_{i} \Rightarrow u_{i+1}$, $\mathrm{R}\left(u_{i}\right)=\mathrm{R}\left(u_{i+1}\right)$, and $\overline{\mathrm{R}\left(u_{i}\right)}=\overline{1}$, a contradiction, since $\mathrm{R}$ is semistandard.

Thus $\boldsymbol{w}_{i+1}$ is an ordered restricted biword. Thus by Lemma 11, sRSK and sRSK* are mutual inverses on $\operatorname{RBiw}(L, R) \unlhd$ and $\operatorname{SStd}(L, R)$.

Remark 22. When $\mathscr{X}=\mathscr{Y}=\mathbb{N}$, where $<$ is the usual order on integers and every element is of even parity, the super RSK correspondence of Theorem 21 reduces to the classical RSK correspondence $[\mathrm{K}]$. 
Remark 23. As noted in $\S 1$, the existence of a bijection between the sets in Theorem 21 was proved by Bonetti, Senato, and Venezia [BSV], using a more straightforward insertion algorithm which yields a different bijection than the one in Theorem 21. Our motivation in presenting this new bijection is in the direction of fully generalizing the symmetry property of classical RSK, which we do in $\S 6$.

Remark 24. In [SW, §3], Shimozono and White present a close relative to our super RSK correspondence - the algorithm they use to construct the upper and lower tableaux of an $(\mathscr{X}, \mathscr{Y})$-biword (called in their paper a doubly-colored biword) is very similar in spirit to the super RSK algorithm presented here (see Remark 6). However, they work with the set of all (not just restricted) biwords, and their semistandard tableaux are defined to be row-weak and column-strict for both parities. Consequently, the fact that a bijective correspondence exists between these objects (as noted in [SW, Theorem 22]) can be deduced from classical RSK correspondence, while this is not true of the correspondence in Theorem 21, which involves distinct (and distinctly-sized) sets of combinatorial objects.

Note that $\operatorname{RBiw}(L, R)^{\unlhd}$ is a set of orbit representatives for $\operatorname{RBiw}(L, R)$ under the action of the symmetric group $\mathfrak{S}_{k}$. For $\boldsymbol{w} \in \operatorname{RBiw}(L, R)$, write $\boldsymbol{w}^{\unlhd}$ for the unique element of $\operatorname{RBiw}(L, R)^{\unlhd}$ which belongs to the $\mathfrak{S}_{k}$-orbit of $\boldsymbol{w}$. By precomposing with the function $\boldsymbol{w} \mapsto \boldsymbol{w}^{\unlhd}$, we may extend $\operatorname{sRSK}$ to a function $\operatorname{sRSK}: \operatorname{RBiw}(L, R) \rightarrow \operatorname{SStd}(L, R)$ which is constant on $\mathfrak{S}_{k}$-orbits.

\section{Symmetry}

In this section we prove that the super RSK algorithm defined in $\S 5.2$ satisfies the symmetry property that holds for the classical RSK algorithm. In this section we assume that $(L, R)$ is an $(\mathscr{X}, \mathscr{Y})$-content pair of length $k$.

\subsection{Inversion}

Let $\boldsymbol{w}=\left(\left(x_{1}, y_{1}\right), \ldots,\left(x_{k}, y_{k}\right)\right) \in \operatorname{RBiw}(L, R)^{\unlhd}$. Then there is a unique biword $\boldsymbol{w}^{\text {inv }} \in$ $\operatorname{RBiw}(R, L)^{\unlhd}$ which consists of the biletters $\left(y_{1}, x_{1}\right), \ldots,\left(y_{k}, x_{k}\right)$. I.e., we construct $\boldsymbol{w}^{\text {inv }}$ by swapping the entries of the biletters in $\boldsymbol{w}$, then reordering the biletters according to the ordering on biletters. We refer to $\boldsymbol{w}^{\text {inv }}$ as the inversion of $\boldsymbol{w}$. For $(\mathrm{L}, \mathrm{R}) \in \operatorname{Tab}(L, R)$, write $(\mathrm{L}, \mathrm{R})^{\mathrm{inv}}:=(\mathrm{R}, \mathrm{L}) \in \operatorname{Tab}(R, L)$.

\subsection{Standardizing biwords}

We will say a biword is standard if no letter occurring in the biword has multiplicity greater than one. For a multiset $L$ of letters in $\mathscr{X}$, we define

$$
L^{\bullet}=\left\{x^{(i)} \in \mathscr{X}^{\bullet} \mid x \in \mathscr{X}, 1 \leqslant i \leqslant \operatorname{mult}_{L}(x)\right\},
$$

where $\operatorname{mult}_{L}(x)$ is the multiplicity of $x$ in $L$. 
Definition 25. Let $(L, R)$ be an $(\mathscr{X}, \mathscr{Y})$-content pair of length $k$. Let $\boldsymbol{w} \in \operatorname{RBiw}(L, R)^{\unlhd}$. We construct a related biword in $\operatorname{RBiw}\left(L^{\bullet}, R^{\bullet}\right)^{\unlhd}$ as follows.

Label the distinct elements of $L$ such that $x_{1} \prec x_{2} \prec \cdots \prec x_{s}$, and label the distinct elements of $R$ such that $y_{1} \prec y_{2} \prec \cdots \prec y_{t}$. Let $\ell_{i, j}$ be the multiplicity of $\left(x_{i}, y_{j}\right)$ in $\boldsymbol{w}$. Then define $\boldsymbol{w}^{\bullet}$ to be the unique biword in $\operatorname{RBiw}\left(L^{\bullet}, R^{\bullet}\right)^{\unlhd}$ which consists of the biletters

$$
\left(x_{i}^{\left(\ell_{i, 1}+\cdots+\ell_{i, j-1}+m\right)}, y_{j}^{\left(\ell_{1, j}+\cdots+\ell_{i-1, j}+m\right)}\right) \text { for } i \in[1, s], j \in[1, t], m \in\left[1, \ell_{i, j}\right], \bar{y}_{j}=\overline{0},
$$

and

$$
\left(x_{i}^{\left(\ell_{i, 1}+\cdots+\ell_{i, j-1}+m\right)}, y_{j}^{\left(\ell_{1, j}+\cdots+\ell_{i, j}+1-m\right)}\right) \text { for } i \in[1, s], j \in[1, t], m \in\left[1, \ell_{i, j}\right], \bar{y}_{j}=\overline{1} .
$$

We call $\boldsymbol{w}^{\bullet}$ the $\bullet$-standardization of $\boldsymbol{w}$.

Note that by construction, $\boldsymbol{w}^{\bullet}$ is a standard biword. Define $\operatorname{SRBiw}\left(L^{\bullet}, R^{\bullet}\right)^{\unlhd}$ as the set of standard restricted biwords in $\operatorname{RBiw}\left(L^{\bullet}, R^{\bullet}\right)^{\unlhd}$. Let $\bullet: \operatorname{RBiw}(L, R)^{\unlhd} \rightarrow \operatorname{SRBiw}\left(L^{\bullet}, R^{\bullet}\right)^{\unlhd}$ be the map defined by $\boldsymbol{w} \mapsto \boldsymbol{w}^{\bullet}$. Let $\hat{\bullet}: \operatorname{SRBiw}\left(L^{\bullet}, R^{\bullet}\right)^{\unlhd} \rightarrow \operatorname{Biw}(L, R)$ be given by 'forgetting superscripts'. By definition of the orders on biletters we have that $\hat{\bullet} \circ \bullet$ is the identity on $\operatorname{RBiw}(L, R)^{\unlhd}$.

Lemma 26. For $\boldsymbol{w} \in \operatorname{RBiw}(L, R)^{\unlhd}$, we have $\left(\boldsymbol{w}^{\bullet}\right)^{\mathrm{inv}}=\left(\boldsymbol{w}^{\mathrm{inv}}\right)^{\bullet}$.

Proof. If $\ell_{i, j}$ is the multiplicity of $\left(x_{i}, y_{j}\right)$ in $\boldsymbol{w}$, then $\ell_{i, j}$ is the multiplicity of $\left(y_{j}, x_{i}\right)$ in $\boldsymbol{w}^{\text {inv }}$. Then $\left(\boldsymbol{w}^{\bullet}\right)^{\text {inv }}$ consists of the biletters

$$
\left(y_{j}^{\left(\ell_{1, j}+\cdots+\ell_{i-1, j}+m\right)}, x_{i}^{\left(\ell_{i, 1}+\cdots+\ell_{i, j-1}+m\right)}\right) \text { for } i \in[1, s], j \in[1, t], m \in\left[1, \ell_{i, j}\right], \bar{y}_{j}=\overline{0},
$$

and

$$
\left(y_{j}^{\left(\ell_{1, j}+\cdots+\ell_{i, j}+1-m\right)}, x_{i}^{\left(\ell_{i, 1}+\cdots+\ell_{i, j-1}+m\right)}\right) \text { for } i \in[1, s], j \in[1, t], m \in\left[1, \ell_{i, j}\right], \bar{y}_{j}=\overline{1} .
$$

while $\left(\boldsymbol{w}^{\text {inv }}\right)^{\bullet}$ consists of the biletters

$$
\left(y_{j}^{\left(\ell_{1, j}+\cdots+\ell_{i-1, j}+m\right)}, x_{i}^{\left(\ell_{i, 1}+\cdots+\ell_{i, j-1}+m\right)}\right) \text { for } j \in[1, t], i \in[1, s], m \in\left[1, \ell_{i, j}\right], \bar{x}_{i}=\overline{0},
$$

and

$$
\left(y_{j}^{\left(\ell_{1, j}+\cdots+\ell_{i-1, j}+m\right)}, x_{i}^{\left(\ell_{i, 1}+\cdots+\ell_{i, j}+1-m\right)}\right) \text { for } j \in[1, t], i \in[1, s], m \in\left[1, \ell_{i, j}\right], \bar{x}_{i}=\overline{1} .
$$

Assume that $\left(y_{j}^{(a)}, x_{i}^{(b)}\right)$ is a biletter of $\left(\boldsymbol{w}^{\bullet}\right)^{\text {inv }}$, for some $j \in[1, t], i \in[1, s]$, and $a, b \in \mathbb{Z}_{>0}$. We will show that $\left(y_{j}^{(a)}, y_{i}^{(b)}\right)$ is a biletter in $\left(\boldsymbol{w}^{\text {inv }}\right)^{\bullet}$ as well. We consider three cases:

(a) Assume that $\bar{y}_{j}+\bar{x}_{i}=\overline{1}$. Then $\ell_{i, j}=1$ since $\boldsymbol{w}$ is a restricted biword. Then $a=\ell_{1, j}+\cdots+\ell_{i, j}$ and $b=\ell_{i, 1}+\cdots+\ell_{i, j}$, so the claim follows. 
(b) Assume that $\bar{y}_{j}=\bar{x}_{i}=\overline{0}$. Then $a=\ell_{1, j}+\cdots+\ell_{i-1, j}+m$ and $b=\ell_{i, 1}+\cdots+\ell_{i, j-1}+m$, for some $m \in\left[1, \ell_{i, j}\right]$, so the claim follows.

(c) Assume that $\bar{y}_{j}=\bar{x}_{i}=\overline{1}$. Then $a=\ell_{1, j}+\cdots+\ell_{i, j}+1-m$ and $b=\ell_{i, 1}+\cdots+\ell_{i, j-1}+m$, for some $m \in\left[1, \ell_{i, j}\right]$. But then, setting $n=\ell_{i, j}-m+1$, we have that $n \in\left[1, \ell_{i, j}\right]$, and $a=\ell_{1, j}+\cdots+\ell_{i-1, j}+n$ and $b=\ell_{i, 1}+\cdots+\ell_{i, j}+1-n$, so the claim follows.

Then in any case, every biletter of $\left(\boldsymbol{w}^{\bullet}\right)^{\text {inv }}$ appears in $\left(\boldsymbol{w}^{\text {inv }}\right)^{\bullet}$. Since the letters in $\boldsymbol{w}^{\bullet}$ appear with multiplicity one, and the biwords $\left(\boldsymbol{w}^{\bullet}\right)^{\text {inv }}$ and $\left(\boldsymbol{w}^{\text {inv }}\right) \bullet$ are ordered and have the same length, this completes the proof of the lemma.

We extend the 'forget superscripts' map $\hat{\bullet}: \mathscr{X}^{\bullet} \rightarrow \mathscr{X}$ to a map $\hat{\bullet}: \operatorname{SStd}\left(L^{\bullet}, R^{\bullet}\right) \rightarrow$ $\operatorname{Tab}(L, R)$ via 'forgetting superscripts' of all entries in the tableaux.

Lemma 27. For $\boldsymbol{w} \in \operatorname{RBiw}(L, R)^{\unlhd}$ we have $\hat{\bullet}\left(\operatorname{sRSK}\left(\boldsymbol{w}^{\bullet}\right)\right)=\operatorname{sRSK}(\boldsymbol{w})$.

Proof. Recall from Definition 25 that we label the distinct elements of $L$ such that $x_{1} \prec$ $x_{2} \prec \cdots \prec x_{s}$, and label the distinct elements of $R$ such that $y_{1} \prec y_{2} \prec \cdots \prec y_{t}$. Assume that $\left(x_{i}^{(a)}, y_{j}^{(b)}\right) \triangleleft\left(x_{i}^{(c)}, y_{j^{\prime}}^{(d)}\right)$ are biletters in $\boldsymbol{w}^{\bullet}$. We first prove that $x_{i}^{(a)} \prec x_{i}^{(c)}$ if and only if $\bar{x}_{i}+\bar{y}_{j^{\prime}}=\overline{0}$. There are four cases to consider:

(a) Assume that $j<j^{\prime}$. Then we have $y_{j} \prec y_{j^{\prime}}$ and $y_{j}^{(b)}<y_{j^{\prime}}^{(d)}$, which implies that $y_{j}<y_{j^{\prime}}$. Then $\bar{y}_{j^{\prime}}=\overline{0}$. By the definition of the $\bullet$-standardization $\boldsymbol{w}^{\bullet}$, we have, for some $m \in\left[1, \ell_{i, j}\right], n \in\left[1, \ell_{i, j^{\prime}}\right]$,

$$
a=\ell_{i, 1}+\cdots+\ell_{i, j-1}+m<\ell_{i, 1}+\cdots+\ell_{i, j-1}+\cdots+\ell_{i, j^{\prime}-1}+n=c .
$$

Thus $x_{i}^{(a)}<x_{i}^{(c)}$. The claim follows.

(b) Assume that $j>j^{\prime}$. Then we have $y_{j} \succ y_{j^{\prime}}$ and $y_{j}^{(b)}<y_{j^{\prime}}^{(d)}$, which implies that $y_{j}>y_{j^{\prime}}$. Then $\bar{y}_{j^{\prime}}=\overline{1}$. Then by the definition of the $\bullet$-standardization $\boldsymbol{w}^{\bullet}$, we have, for some $m \in\left[1, \ell_{i, j}\right], n \in\left[1, \ell_{i, j^{\prime}}\right]$,

$$
a=\ell_{i, 1}+\cdots+\ell_{i, j^{\prime}-1}+\cdots+\ell_{i, j-1}+m>\ell_{i, 1}+\cdots+\ell_{i, j^{\prime}-1}+n=c .
$$

Thus $x_{i}^{(a)}>x_{i}^{(c)}$. The claim follows.

(c) Assume that $j=j^{\prime}$ and $\bar{y}_{j^{\prime}}=\overline{0}$. Then $b<d$ since $y_{j}^{(b)}=y_{j^{\prime}}^{(b)}<y_{j^{\prime}}^{(d)}$, and we have, for some $m, n \in\left[1, \ell_{i, j^{\prime}}\right]$,

$$
\ell_{1, j^{\prime}}+\cdots+\ell_{i-1, j^{\prime}}+m=b<d=\ell_{1, j^{\prime}}+\cdots+\ell_{i-1, j^{\prime}}+n,
$$

so $m<n$. Then we have

$$
a=\ell_{i, 1}+\cdots+\ell_{i, j^{\prime}-1}+m<\ell_{i, 1}+\cdots+\ell_{i, j^{\prime}-1}+n=c,
$$

so $x_{i}^{(a)}<x_{i}^{(c)}$. The claim follows. 
(d) Assume that $j=j^{\prime}$ and $\bar{y}_{j^{\prime}}=\overline{1}$. Then $b<d$ since $y_{j}^{(b)}=y_{j^{\prime}}^{(b)}<y_{j^{\prime}}^{(d)}$, and we have, for some $m, n \in\left[1, \ell_{i, j^{\prime}}\right]$,

$$
\ell_{1, j^{\prime}}+\cdots+\ell_{i, j^{\prime}}+1-m=b<d=\ell_{1, j^{\prime}}+\cdots+\ell_{i, j^{\prime}}+1-n,
$$

so $m>n$. Then we have

$$
a=\ell_{i, 1}+\cdots+\ell_{i, j^{\prime}-1}+m>\ell_{i, 1}+\cdots+\ell_{i, j^{\prime}-1}+n=c,
$$

so $x_{i}^{(a)}>x_{i}^{(c)}$. The claim follows.

Thus in any case, the claim follows.

Let $\mathrm{T}_{\boldsymbol{w}}^{i}$ (resp. $\mathrm{T}_{\boldsymbol{w}}^{i}$ ) be the $i$ th insertion tableaux in the Super RSK algorithm applied to $\boldsymbol{w}$ (resp. $\boldsymbol{w}^{\bullet}$ ), and let $a_{\boldsymbol{w}}^{i}$ (resp. $a_{\boldsymbol{w}}^{i} \boldsymbol{\bullet}$ ) be the added node of this insertion, using notation in $§ 5.2$. By induction, assume that $\hat{\bullet}\left(\mathrm{T}_{\boldsymbol{w}}^{i} \bullet\right)$ is a $\bullet$-standardization of $\mathrm{T}_{\boldsymbol{w}}^{i}$, and $a_{\boldsymbol{w}}^{i} \boldsymbol{\bullet}=a_{\boldsymbol{w}}^{i}$, for all $i<n$. If $\left(x_{i}^{(c)}, y_{j^{\prime}}^{(d)}\right)$ is the $n$th biletter in $\boldsymbol{w}^{\bullet}$, then $\left(x_{i}, y_{j^{\prime}}\right)$ is the $n$th biletter in $\boldsymbol{w}$. If $\bar{y}_{j^{\prime}}+\bar{x}_{i}=\overline{0}$, then, by the above claim, we have $x_{i}^{(c)} \succ z$ for every $z \in \mathrm{T}_{\boldsymbol{w}^{\bullet}}^{n-1}$ such that $\hat{\bullet}(z)=x_{i}$. On the other hand if $\bar{y}_{j^{\prime}}+\bar{x}_{i}=\overline{1}$, then, by the above claim, we have $x_{i}^{(c)} \prec z$ for every $z \in \mathrm{T}_{\boldsymbol{w}^{\bullet}}^{n-1}$ such that $\hat{\bullet}(z)=x_{i}$. Therefore by Lemma 14, it follows that $\mathrm{T}_{\boldsymbol{w}}^{n} \boldsymbol{\bullet}=\left(\mathrm{T}_{\boldsymbol{w}^{\bullet}}^{n-1} \stackrel{\bar{y}_{j^{\prime}}}{\longleftarrow} x_{i}^{(c)}\right)$ is a $\bullet$-standardization of $\left(\mathrm{T}_{\boldsymbol{w}}^{n-1} \stackrel{\bar{y}_{j^{\prime}}}{\longleftarrow} x_{i}\right)=\mathrm{T}_{\boldsymbol{w}}^{n}$, and $a_{\boldsymbol{w}}^{n} \boldsymbol{\bullet}=a_{\boldsymbol{w}}^{n}$, as desired. Thus $\hat{\bullet}\left(\mathrm{T}_{\boldsymbol{w}^{\bullet}}\right)=\mathrm{T}_{\boldsymbol{w}}$ and $\hat{\bullet}\left(\mathrm{T}^{\boldsymbol{\bullet}}\right)=\mathrm{T}^{\boldsymbol{w}}$, so $\hat{\bullet}\left(\operatorname{sRSK}\left(\boldsymbol{w}^{\bullet}\right)\right)=\operatorname{sRSK}(\boldsymbol{w})$, proving the lemma.

\subsection{Symmetry}

As noted in Remark 24, Shimizono and White [SW] define a super-analogue of the RSK algorithm which is identical to the super RSK algorithm presented here when restricted to standard biwords. Thus their symmetry result proves a special case of the symmetry of the sRSK map:

Lemma 28. If $\boldsymbol{w} \in \operatorname{RBiw}(L, R)^{\unlhd}$ is a standard biword, then we have

$$
\operatorname{sRSK}\left(\boldsymbol{w}^{\mathrm{inv}}\right)=(\operatorname{sRSK}(\boldsymbol{w}))^{\mathrm{inv}} .
$$

Proof. This follows from [SW, Theorem 21(3),(6)].

Now we extend this result to the general case.

Theorem 29. The following is a commuting diagram: 


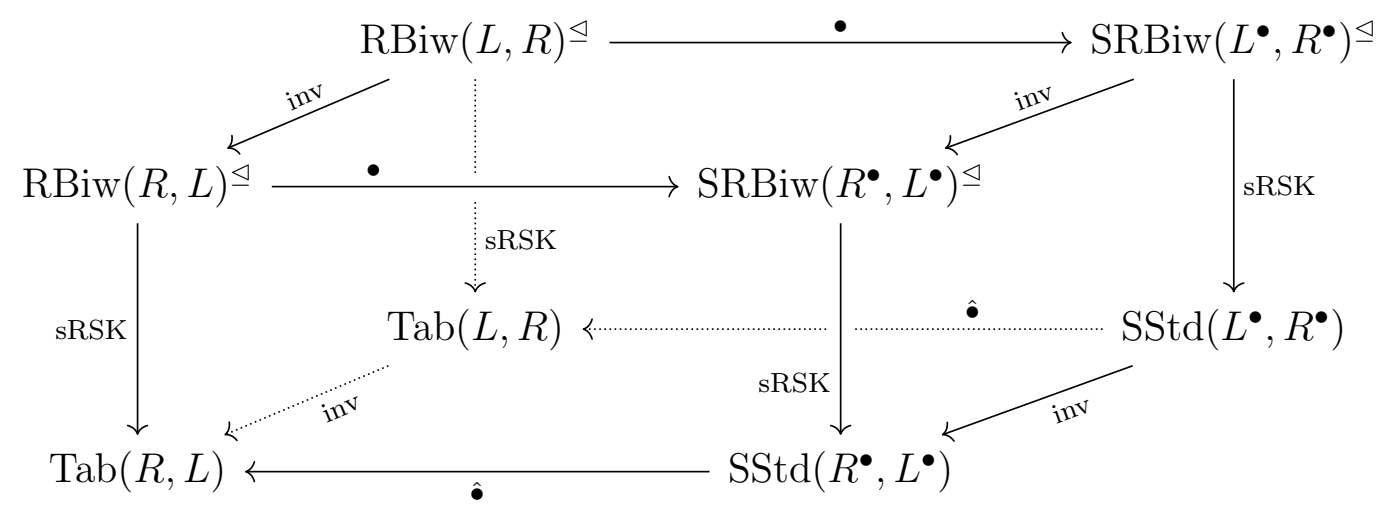

Proof. The top face commutes by Lemma 26. The bottom face commutes since 'forgetting superscripts' then swapping tableaux clearly yields the same result as swapping tableaux and then 'forgetting superscripts'. The front and back faces commute by Lemma 27. The right face commutes by Lemma 28. Thus we have

$$
\begin{aligned}
\mathrm{sRSK} \circ \mathrm{inv} & =\hat{\bullet} \circ \mathrm{sRSK} \circ \bullet \circ \mathrm{inv}=\hat{\bullet} \circ \mathrm{sRSK} \circ \operatorname{inv} \circ \bullet \\
& =\hat{\bullet} \circ \operatorname{inv} \circ \operatorname{sRSK} \circ \bullet=\operatorname{inv} \circ \hat{\bullet} \circ \operatorname{sRSK} \circ \bullet=\operatorname{inv} \circ \mathrm{sRSK},
\end{aligned}
$$

so the left face commutes, proving the theorem.

Corollary 30 (Super RSK symmetry). For all $\boldsymbol{w} \in \operatorname{RBiw}(L, R)^{\unlhd}$ we have

$$
\operatorname{sRSK}\left(\boldsymbol{w}^{\mathrm{inv}}\right)=(\operatorname{sRSK}(\boldsymbol{w}))^{\mathrm{inv}} .
$$

Example 31. As in Example 20, take the alphabet

$$
\mathscr{X}=\mathscr{Y}=\{\hat{1}<1<\hat{2}<2<\hat{3}<3\},
$$

where odd parity letters are indicated by carets, and the restricted biword

$$
\boldsymbol{w}=((\hat{3}, \hat{1}),(1, \hat{2}),(2,2),(3,2),(\hat{3}, \hat{3}),(\hat{3}, \hat{3}),(\hat{2}, 3),(\hat{1}, 3)) .
$$

The inversion of $\boldsymbol{w}$ is

$$
\boldsymbol{w}^{\text {inv }}=((3, \hat{1}),(\hat{2}, 1),(3, \hat{2}),(2,2),(\hat{3}, \hat{3}),(\hat{3}, \hat{3}),(\hat{1}, \hat{3}),(2,3)) .
$$

We have

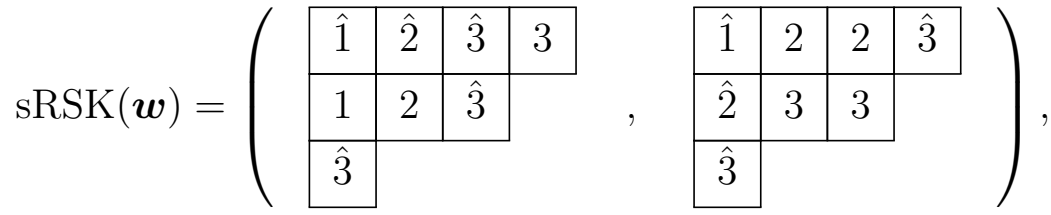

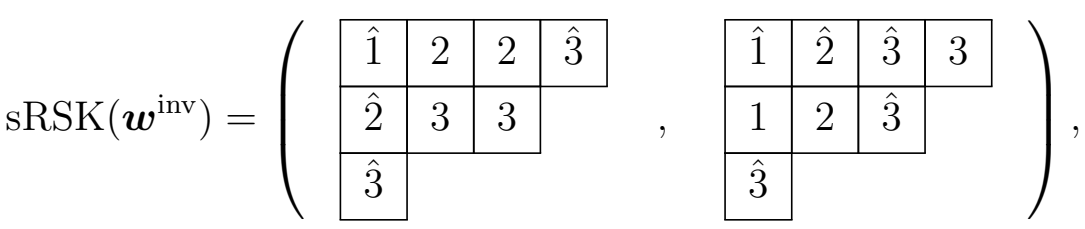


so $\operatorname{sRSK}\left(\boldsymbol{w}^{\mathrm{inv}}\right)=\operatorname{sRSK}(\boldsymbol{w})^{\mathrm{inv}}$, as expected.

By way of comparison, consider the super-RSK algorithm (label it sRSK $\mathrm{BSV}$ to differentiate it from the algorithm in this paper) defined by Bonetti, Senato and Venezia [BSV]. When we apply sRSK ${ }_{\mathrm{BSV}} \boldsymbol{w}$ and $\boldsymbol{w}^{\text {inv }}$, we get (after reordering the biletters to agree with their combinatorial setup):

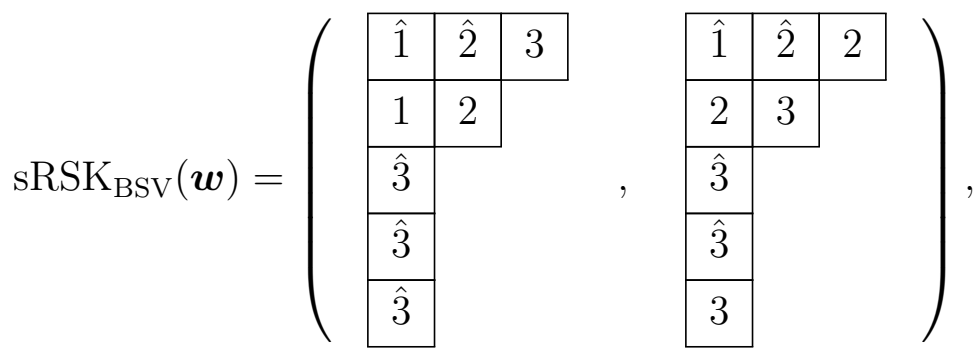

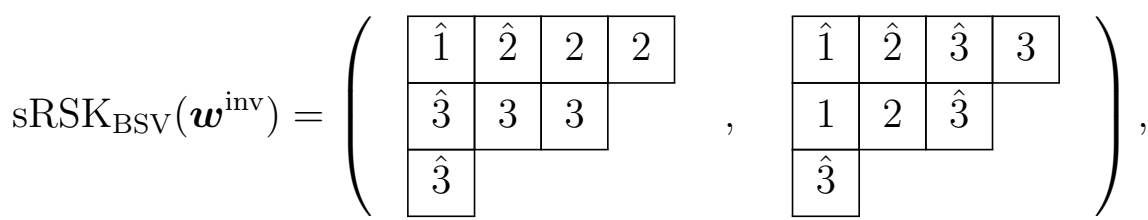

so evidently symmetry does not generally hold for sRSK $\mathrm{BSV}_{\text {. }}$

Finally consider the super-RSK algorithm (label it sRSK ${ }_{\mathrm{SW}}$ ) defined by Shimozono and White $[\mathrm{SW}]$. When we apply $\mathrm{sRSK}_{\mathrm{SW}}$ to $\boldsymbol{w}$ and $\boldsymbol{w}^{\text {inv }}$, we get (again after reordering biletters):

$$
\begin{aligned}
& \operatorname{sRSK}_{\mathrm{SW}}(\boldsymbol{w})=\left(\begin{array}{|l|l|l|l|l|}
\hat{1} & \hat{2} & \hat{3} & \hat{3} & \hat{3} \\
\cline { 1 - 6 } & 2 & 3 & &
\end{array}, \begin{array}{|l|l|l|l|l|l|}
\hat{1} & 2 & 2 & \hat{3} & \hat{3} \\
\hline \hat{2} & 3 & 3 & &
\end{array}\right), \\
& \operatorname{sRSK}_{\mathrm{SW}}\left(\boldsymbol{w}^{\text {inv }}\right)=\left(\begin{array}{l|l|l|l|l|}
\hat{1} & 2 & 2 & \hat{3} & \hat{3} \\
\hline \hat{2} & 3 & 3 & &
\end{array}, \quad \begin{array}{ll|l|l|l|l|}
\hat{1} & \hat{2} & \hat{3} & \hat{3} & \hat{3} \\
\hline 1 & 2 & 3 & &
\end{array}\right),
\end{aligned}
$$

so $\operatorname{sRSK}_{\mathrm{SW}}\left(\boldsymbol{w}^{\mathrm{inv}}\right)=\operatorname{sRSK}_{\mathrm{SW}}(\boldsymbol{w})^{\text {inv }}$, as expected, given [SW, Theorem 21]. Note however that the tableaux output by the $\mathrm{sRSK}_{\mathrm{SW}}$ algorithm are row-weak and column-strict with respect to both parities, a different flavor of 'semistandard' than the notion defined in this paper.

\section{Acknowledgements}

The author would like to thank Alexander Kleshchev, who originally suggested the topic of the paper as an approach to a representation theoretic problem, and provided helpful suggestions. The author would also like to thank Scott Cook for numerous fruitful discussions. 


\section{References}

[BSV] F. Bonetti, D. Senato, and A. Venezia. The Robinson-Schensted correspondence for the fourfold algebra. Boll. Unione Mat. Ital. VII Ser. B 23 (1998) 541-554.

[CPT] S. Clark, Y.N. Peng and S.K. Thamrongpairoj, Super tableaux and a branching rule for the general linear Lie superalgebra, Linear Multilinear A. 63 (2015) 274.

[DR] J. Du and H. Rui, Quantum Schur superalgebras and Kazhdan-Lusztig combinatorics, J. Pure Appl. Algebra 215 (2011), 2715-2737.

[F] W. Fulton, Young tableaux, London Math. Soc. Stud. Texts 35, Cambridge University Press, Cambridge, 1997.

[GRS] F. D. Grosshans, G. C. Rota, J. A. Stein, Invariant theory and superalgebras, Reg. Conf. Ser. Math. 69, (1987)

[H] M. D. Haiman, On mixed insertion, symmetry, and shifted Young tableaux, J. Combin. Theory Ser. A, 50 (1989), pp. 196-225.

[KM] A. Kleshchev and R. Muth, Schurifying quasihereditary algebras, 48 pp., arXiv: 1810.02849

[K] D. Knuth, Permutations, matrices, and generalized Young tableaux, Pacific J. of Math. 34 (1970), pp. 709-727.

[LNS] R. La Scala, V. Nardozza and D. Senato. Super RSK-algorithms and super plactic monoid. Internat. J. Algebra Comput. 16 2, (2006), 377-396.

[MZ] F. Marko, A. N. Zubkov, A note on bideterminants for Schur superalgebras, J. Pure Appl. Algebra, 215 (2011), 2223-2230.

[R] G. de B. Robinson, On the Representations of the Symmetric Group, Amer. J. Math. 60 (1938): 745-760.

[S] C. Schensted, Longest increasing and decreasing subsequences, Canad. J. Math. 13 (1961), 179-191.

[SW] M. Shimozono and D. E. White, A Color-to-Spin Domino Schensted Algorithm, Electron. J. Combin. 8 (2001), \#R21.

[V] G. Viennot, Une forme géométrique de la correspondance de Robinson-Schensted, Combinatoire et représentation du groupe symétrique, Lecture Notes in Math. 579, Springer (1977), 29-58. 